\title{
القدرة التنبؤية للدجة استخلاه مواقع التواصل الاجتماعي ومستوى الصلابة النفسية في الطلاق العاطفي للى عينة من المتزوجات العاملات في الجامعة الأردنية
}

\author{
عبد الله المهايرة و أمل الحسون
}

Doi: //10.47015/17.3.4

تاريخ قبوله: 2020/5/31

تاريخ تسلم البحث: 2020/4/14

\section{Predictive Ability of the Degree of Social Media Websites Use and Level of Psychological Hardness in Emotional Divorce among a Sample of Married Women Working at the University of Jordan}

Abdallah Almahaireh and Amal Alhasoun, the University of Jordan, Jordan.

Abstract: This study aimed to explore the degree of use of social media websites and the levels of psychological hardness and emotional divorce, as well as to identify predictive ability of the degree of social media websites use and level of psychological hardness in emotional divorce among a sample of married women working at the University of Jordan. The sample of the study consisted of (350) married women working at the University of Jordan in the first semester of $2018 / 2019$. To achieve the aims of the study, the researchers developed three measures: A measure of the degree of use of social networking sites, a measure of psychological hardness, and a measure of emotional divorce. The study results showed a high degree of social media use and a medium level of both psychological Hardness and emotional divorce. In addition, the degree of social media use explained (54\%) of emotional divorce, while psychological Hardness explained only (10\%) of it.

(Keywords: Social Networking Websites, Psychological Hardness, Emotional Divorce, Working Married Women)

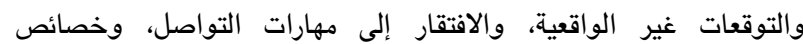

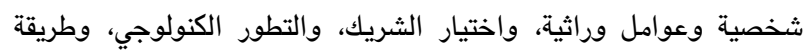

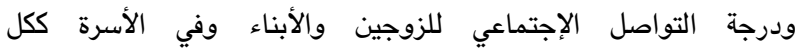

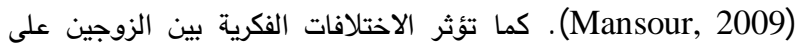

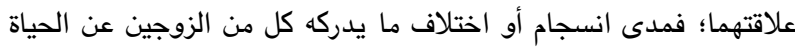

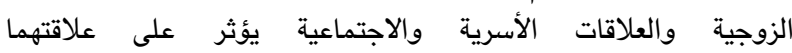

(Fearon, 2003)
ملخص: هدفت الدراسة الحالية إلى الكثف عن درجة استخدام مواقع التواصل

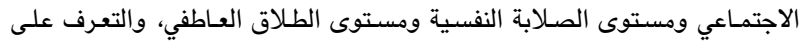

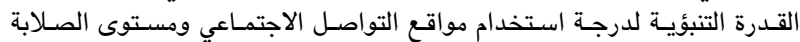

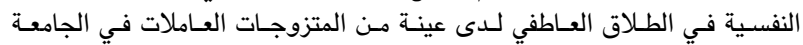

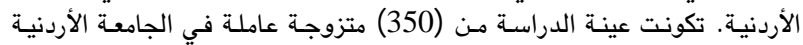

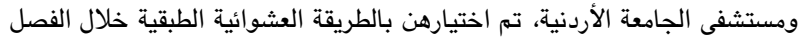

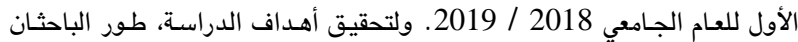

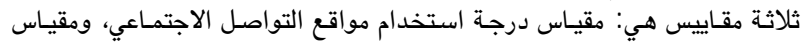

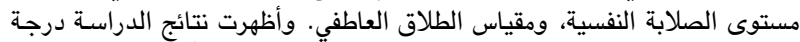

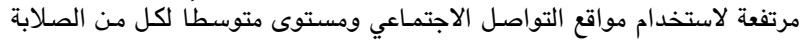

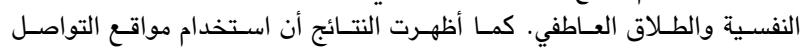

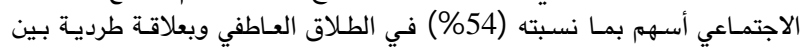

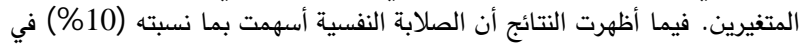
الطلاق العاطفي وبعلاقة طردية بين المتفيرين.

(الكلمـات المفتاحيـة: مواتـع التواصـل الاجتمـاعي، الصـلابة النفسـية، الطـلاق العاطفي، المتزوجات العاملات)

مقدمة: الأسرة هي الخلية الأساسية المكونة للمجتمع، التي لا يمكن

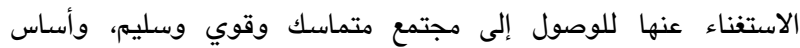

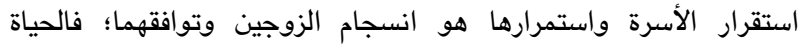

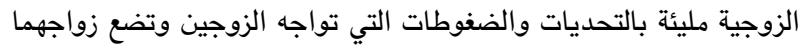

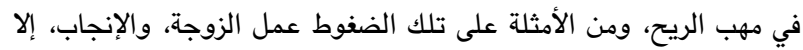

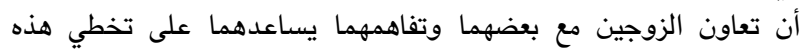
العقبات سويًا، والوصول بالأسرة إلى بر الأمان.

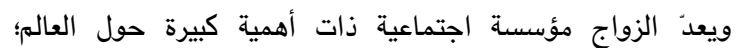

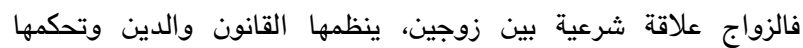
العادات والتقاليد . فهو ليس وظيفة ثابتة، وإنما علاقة متغيرة باستمرار

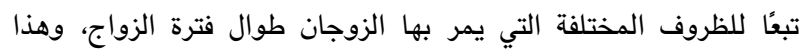

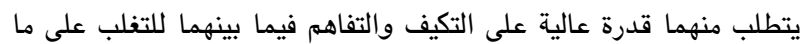

يواجههما من تحديات. (Khoury, 2008).

ويؤثر الزواج في البناء الاجتماعي؛ فمن خلال نماذج التنشئة التي

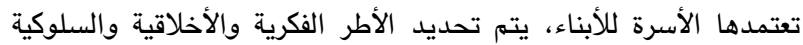
التي تنظم العلاقات بين الأفراد في المجتمع (Theban, 2009) . فالعلاقة الأنة

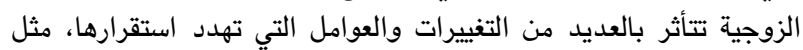

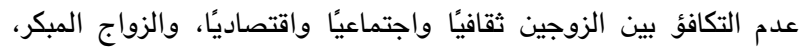

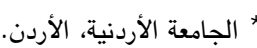
( حقوق الطبع محفوظة لجامعة اليرموك، إربد، الأردن، 2021 الأردن 
خارج المنزل، والرغبة في الانفصال. وهنا تظهر على الطرف الآخر

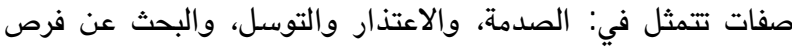

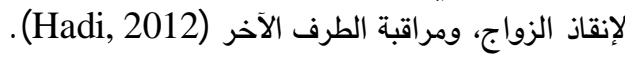

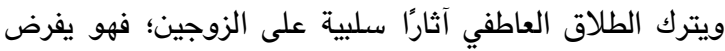

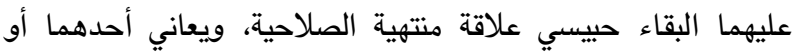

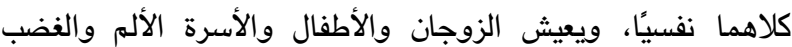

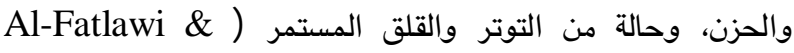

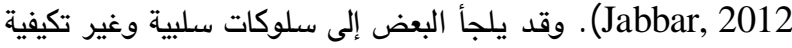
مثل: العدوان والإدمان على الكحول وغيرها من السلوكات السلبية، وإقامة علاقات محرّمة مع طرف آخر، تتؤشر على الهئ الهيار للأسرة والمجتمع (Rodriguez et al., 2009) .

ويشهد العصر الحالي ثورة كبيرة في شتى مجالات الاتصال، مما يترك الأثر الكبير في عملية وشكل وطريقة التواصل الاجتئ فئماعي.

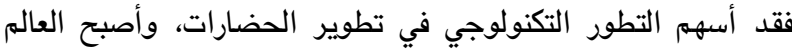

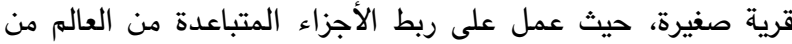

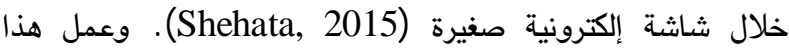

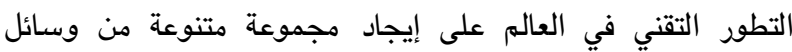
الاتصال، سهّلت الحياة من ناحية ومن ناحية أخرى لا يمكن إنكار التهار

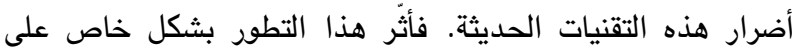

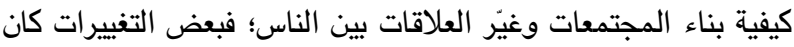

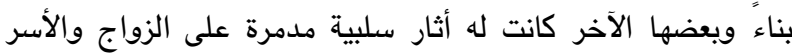
والمجتمعات (Sedghijalal \& Fathi, 2015).

وهناك العديد من العوامل التي تؤثر على العلاقة الزوجية

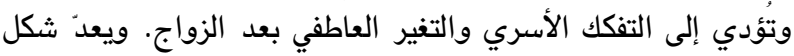

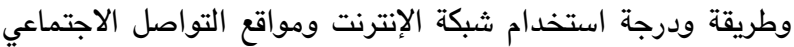

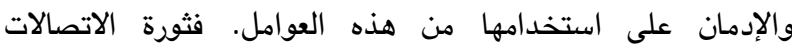

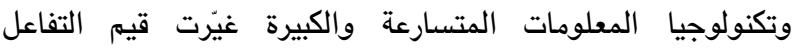

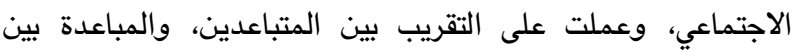

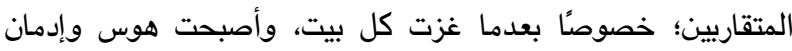

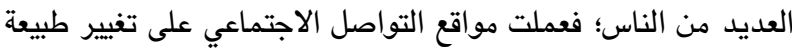

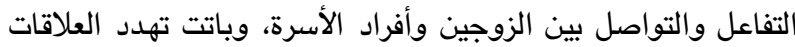

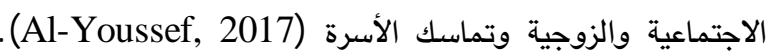

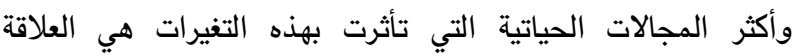

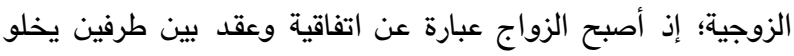

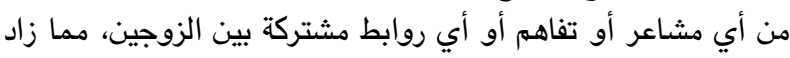
من التحديات التي تحيط بهذه العلاقة (Crow, 2005).

وظهرت العديد من التطييقات مثل اليوتيوب، ومواقع التواصل

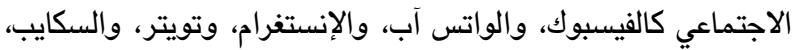

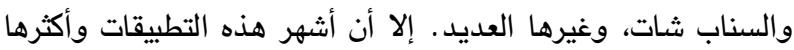

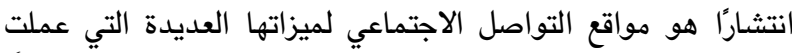

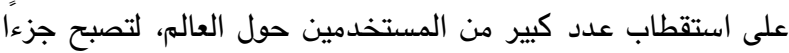

هامًا من حياتهم اليومية (Shakra, 2014).

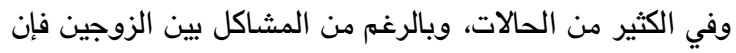

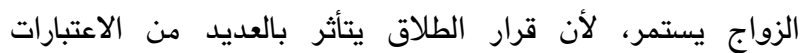

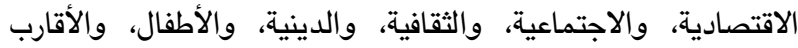

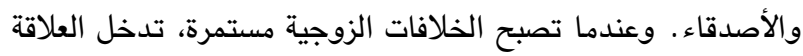

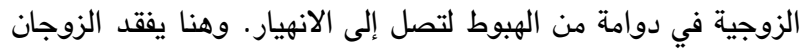

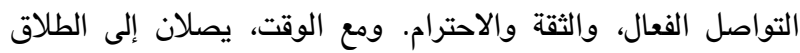

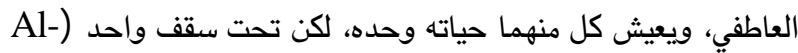

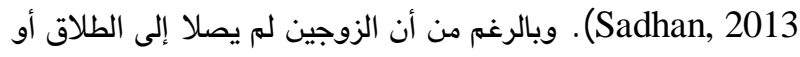

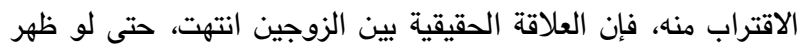

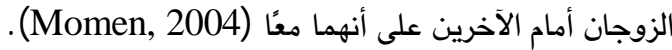
ويعرّت جونسون (Johnson) الطلاق العاطفي بأنه غياب

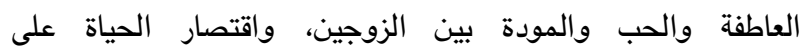
الاحتياجات المادية، واتسام العلاقة بين الزوجين بالنفور، والنزاعات

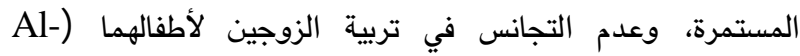
.(Fatlawi \& Jabbar, 2012

والطلاق العاطفي شكل من أشكال الزواج الذي يخلو من الزئ

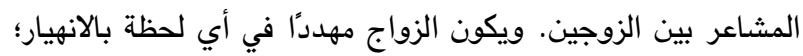

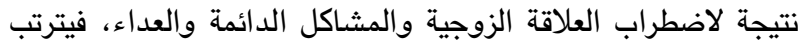

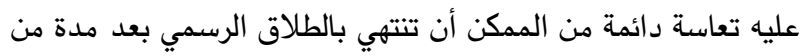

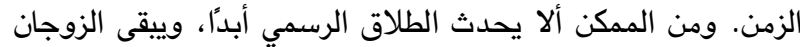

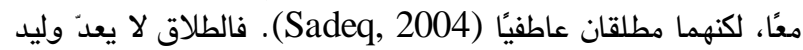

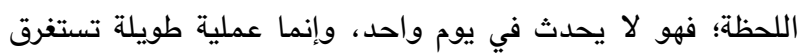

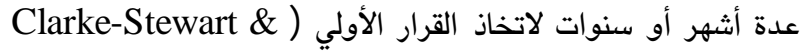

.(Brentano, 2006

ويمر الطلاق العاطفي بسبعة مراحل: المرحلة الأولى (الحلم):

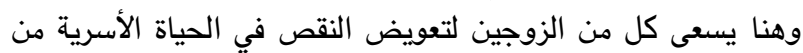

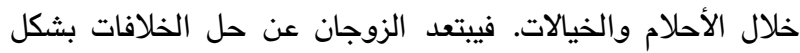

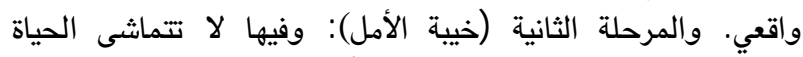

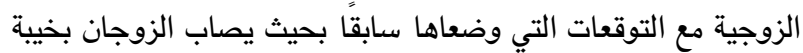

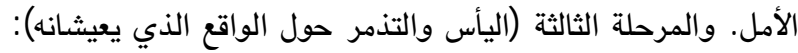

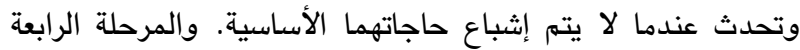

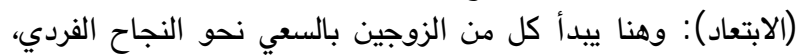

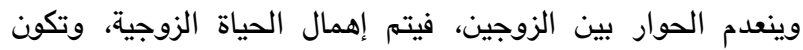

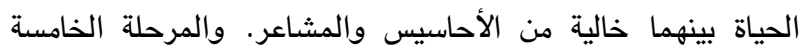

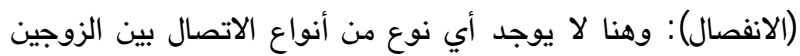

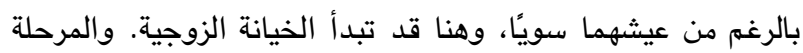

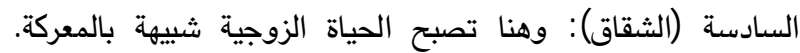

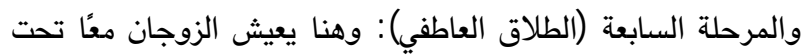

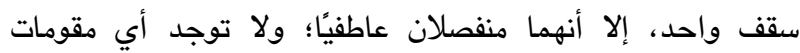
للحياة الزوجية. وتتميز سلوكياتهما بالتباعد والثقاق والات والثمات المشاكل والخلافات الدائمة (Rosperg \& Rosperg, 2002).

ويتسم الزوج المطلق عاطفيًا أو الزوجة المطلقة عاطفيًا

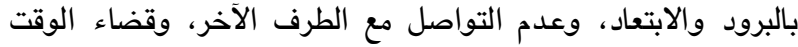




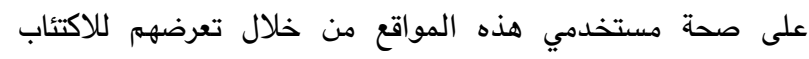

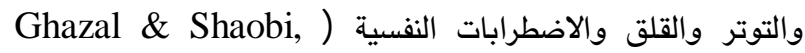
. 2014

وسيطرت مواقع التواصل الاجتماعي في الآونة الأخيرة على

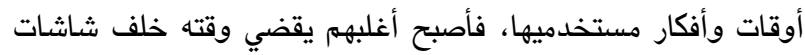

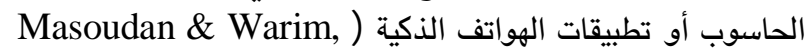

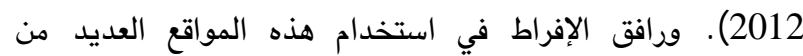

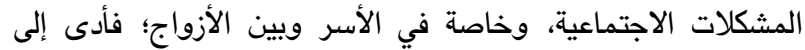

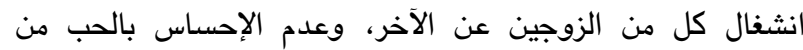

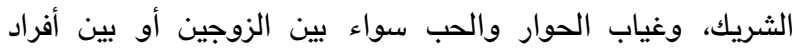

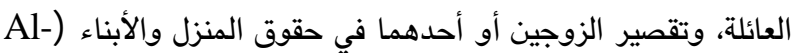
.).Furaiji, 2014

وساعدت التغيرات التي طرأت خلال القرن العشرين من تفيرات

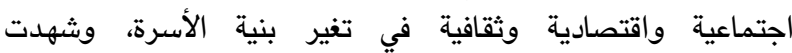
المجتمعات الحديثة تغيرات شملت الجوانب السياسية، والاجتماعية،

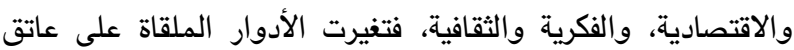

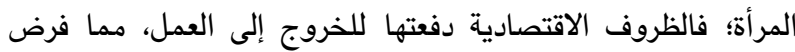

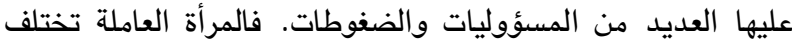

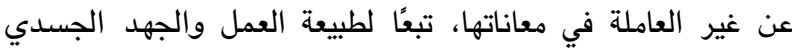
والفكري الذي يتطلبه العمل (Boumediene, 2016). وتمر الحياة الزوجية بالعديد من التحديات والضغوطات؛

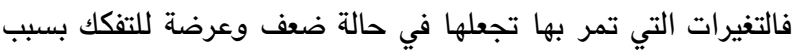

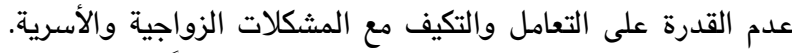

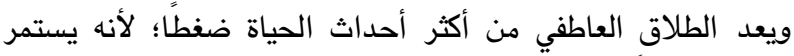

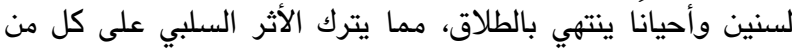

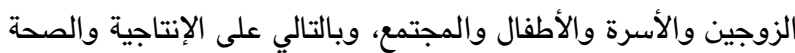
النفية والتكيف (Parvin et al., 2011).

وتم التوصل خلال السنوات الأخيرة إلى أن الضغوطات بحدّ

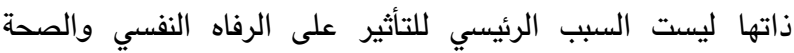

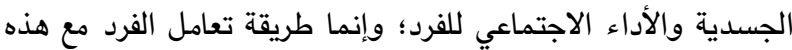

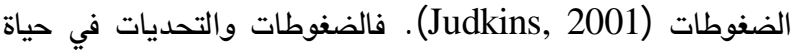

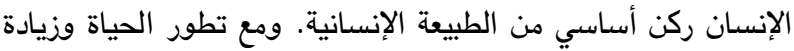

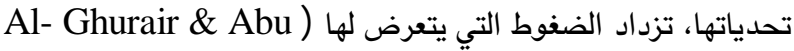

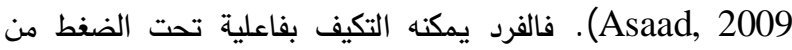
خلال اعتماده على عدة عوامل، كالحدث نفسه، والتقييم المعرفي لهذا

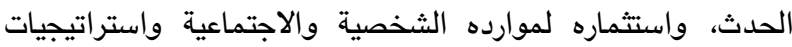
المواجهة التي يلجأ إليها (Judkins, 2001).

ومن خلال العديد من المحاولات التي قامت بها سوزان كوبازا (Susan Cubaza)

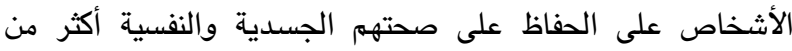
غيرهم، بالرغم من تعرضهم للضفوط، توصلت إلى مفهوم الصلابة الكابة
وتعرف مواتع التواصل الاجتماعي بأنها منظومة من الشبكات

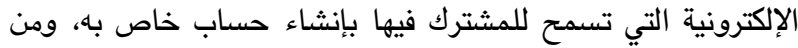

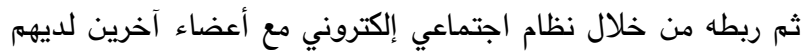

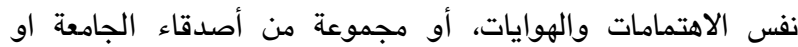
الثانوية (Fadlullah, 2010).

ويبلغ عدد مستخدمي الإنترنت في الأردن حوالي (8) ملايين

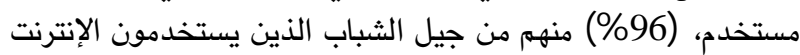
بشكل يومي، فيما بلغ عدد مستخدمي شبكات التواصل الاجتماعي

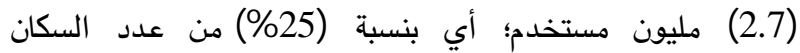

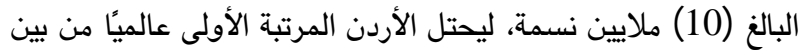

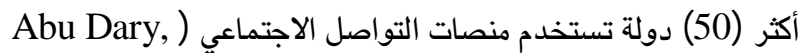

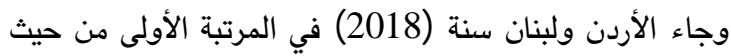

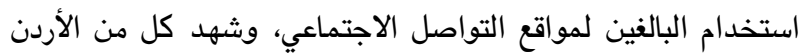

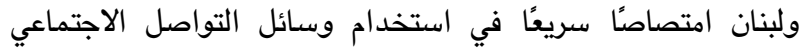
خلال العامين الماضيين؛ فقد زاد استخدام الشبكات الاجتمان الإنماعية

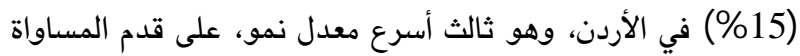

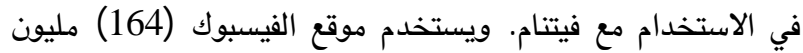

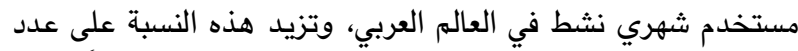

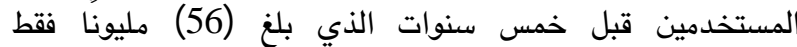

.(Radcliffe \& Bruni, 2019)

وأظهر تقرير عام (2015) أن الأردنيين يستخدمون موقع (89)،

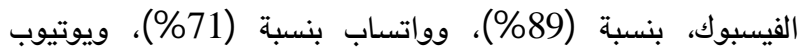

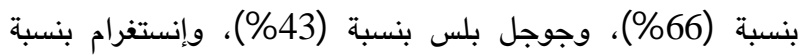

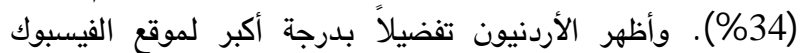

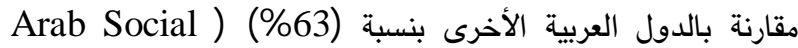
.(Media Report, 2015

وترتب على استخدام هذه المواقع المختلفة العديد من الآثار

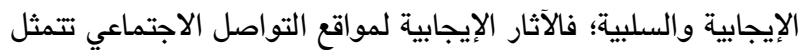

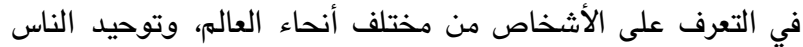

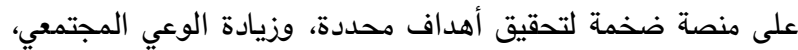

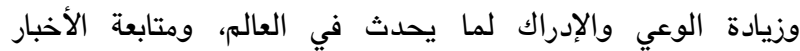
والأحداث العالمية، والعمل والتجارة، وإلفاء الفوارق الطبقية والحدود

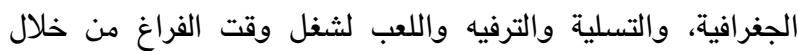
مahmani \& Dhimi, ) مواقع التواصل الاجتماعي المختلفة ولتهن . (2012

أما فيما يتعلق بالآثار السلبية لمواقع التواصل الاجتماعي

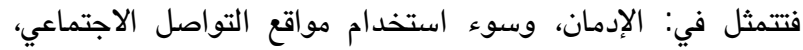

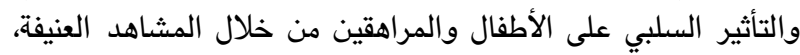
وغزو خصوصية الآخرين، وضعف العلاقات الاجتماعية والزوجية والأسرية (Siddiqui \& Singh, 2016). كما ظهر أيضًا الاستخدام الخاطئ لبيانات وصور المستخدمين الآخرين والتأثير سلبًا 


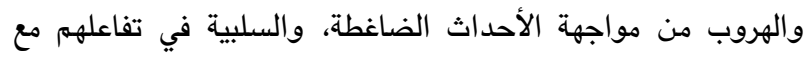

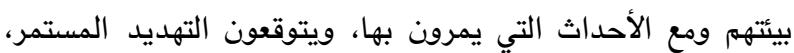

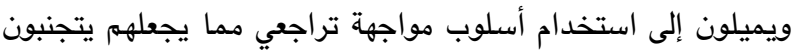
الموقف الضاغط ويبتعدون عنه (Kasemi, 2012).

وقد أجريت العديد من الدراسات حول الطلاق العاطفي

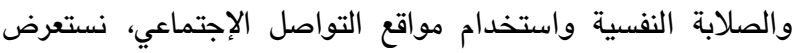

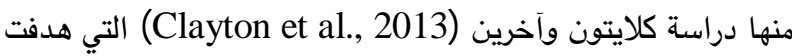

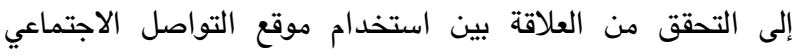

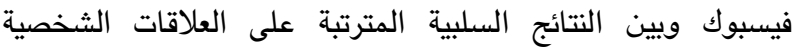

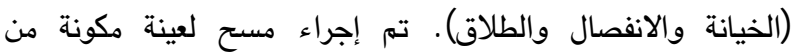

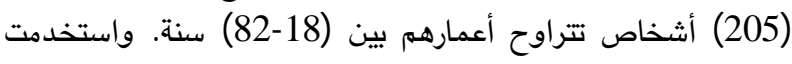

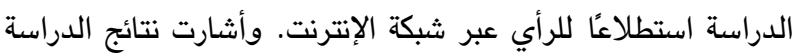

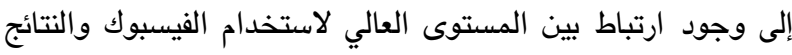

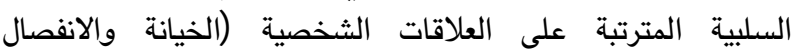

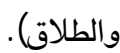

وفي دراسة لنجادات (Nejadat, 2014) حول استخدام المتزوجات العاملات في الجامعات الأردنية للفيسبوك والإشباعات

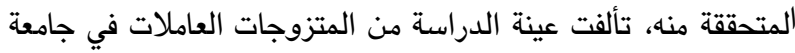

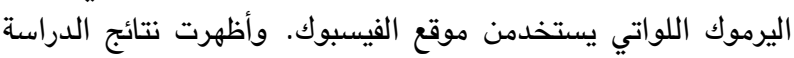

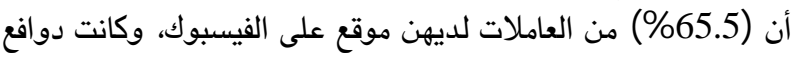

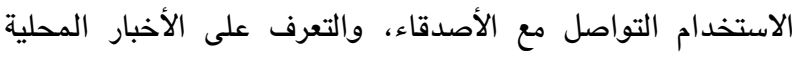

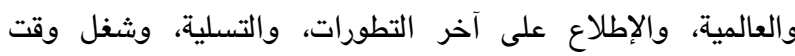
الفراغ.

وقام الثمراني (Al-Shamrani, 2015) بدراسة هدفت إلى الى الكثف عن العلاقة بين شدة استخدام مواقع التواصل الاجتماعي

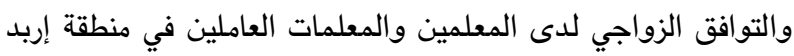

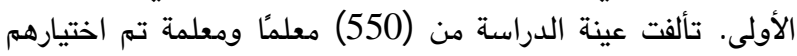

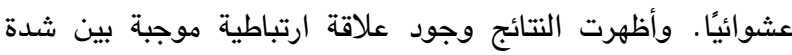

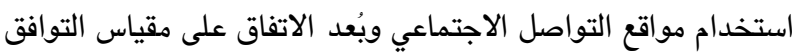

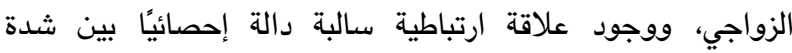

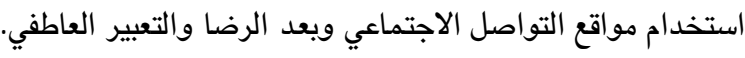

وأجرت شويطر والزقاي ( Shwaiter and Al-Zaqai, )

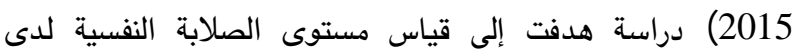

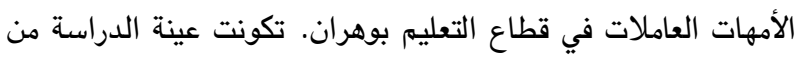

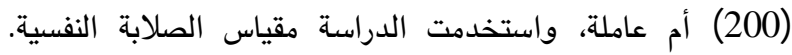
وأظهرت النتائج ارتفاع مستوى الصلابة النفسية لدى الألى الأمهات

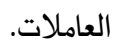

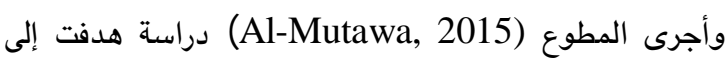

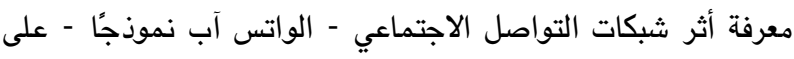

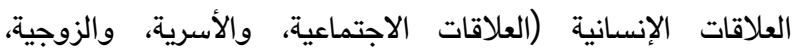

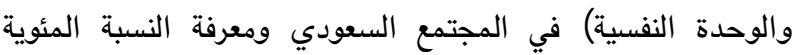
والتكرارات لعادات وأنماط المتزوجين الذين يستخدمون شبكة الواتس ومعة النسية النوية
النفسية، الذي ترى أنه يتضمن ثلاثة أبعاد هي: مكونات الصلابة النفسية (الالتزام، والتحكم، والتحدي) (Yakhlof, 2001). وتعرف الصلابة النفسية بأنها مجموعة من السمات تتمثل في

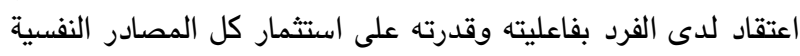

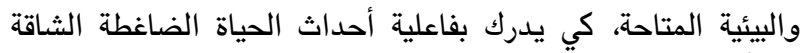

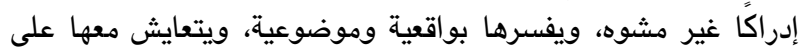
نحو إيجابي. وتتضمن ثلاثة أبعاد هي: التحدي، وماندي، والالتزام، والتحكم

.(Al-Taher, 2016)

ويعرف التحدي بأنه: اعتقاد الشخص أن ما يطرأ من تغيير

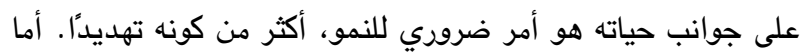

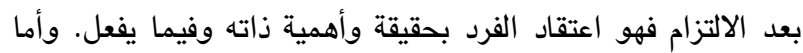

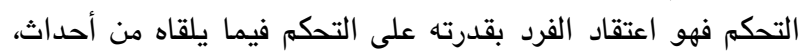
وتحمل المسؤولية الثخصية (Mukhaimar, 2002).

وهذه الأبعاد الثلاثة من وجهة نظر كوبازا هي بنية متعددة

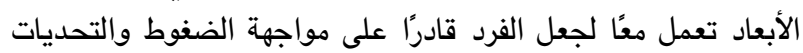

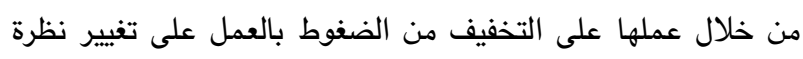

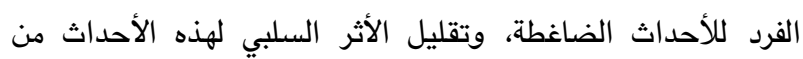

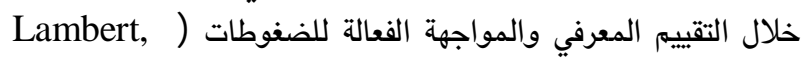
. 2003

ويختلف الأفراد في مستوى الصلابة النفسية لديهم تبعًا

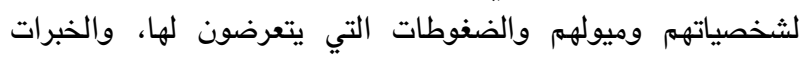

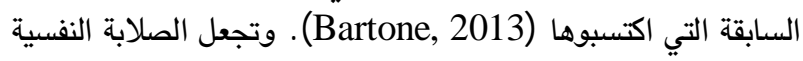

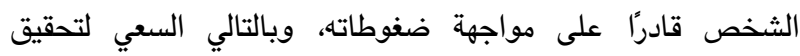

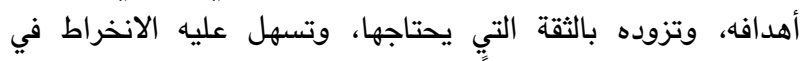

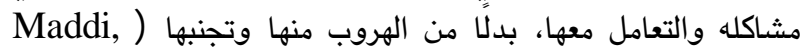

. (2013

والأشخاص الذين يتمتعون بمستوى عال من الصلابة النفسية

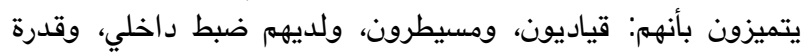

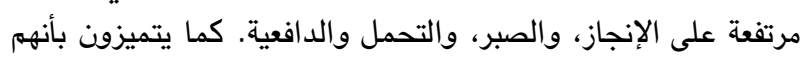

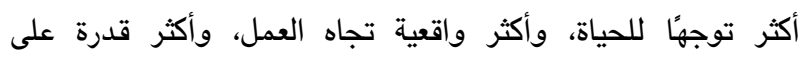

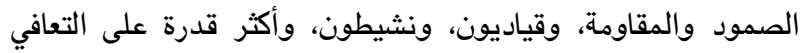
بشكل أسرع من غيرهم (Abu Nada, 2007; Hill, 2007). ويميلون إلى استخدام أسلوب مواجهة تحويلي من خلال تحويل

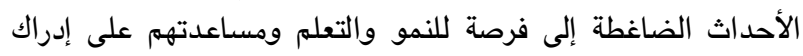

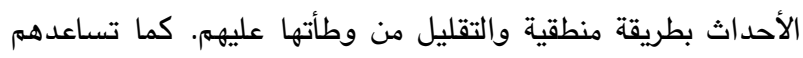

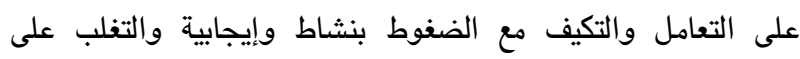

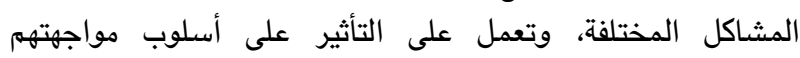
للضغوط والحصول على المساندة الاجتماعية (Rady, 2008). ويتصف الأفراد الذين يتمتعون بمستوى منخفض من الصلابة

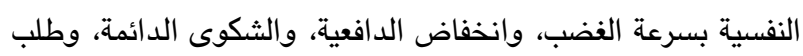

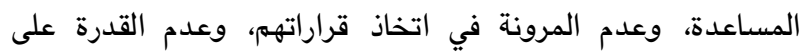
التحكم الذاتي، وعدم القدرة على الصبر، وضعف الأمل في الحياة، 
وأجرى النجداوي (Najdawi, 2018) دراسة هدفت إلى فهم

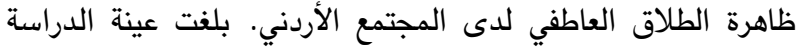

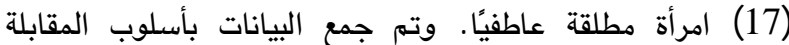
المتعمقة. وأظهرت النتائج وجود طلاق عاطفي في الأردن، وأن أهم الهم الهاب

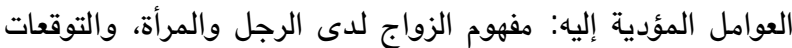

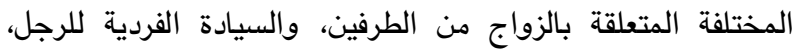

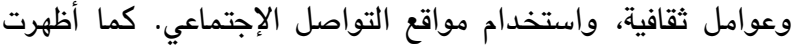

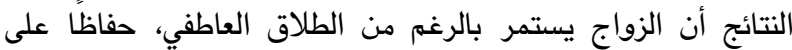

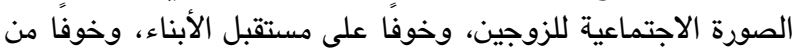

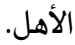

وأجرى الجوازنة (Al-Jawazneh, 2018) دراسة هدفت إلى

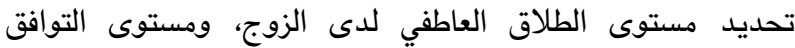
النفسي للأبناء، ومستوى ما يتببأ به الطلاق العاطفي لدى الزئ الزوجين

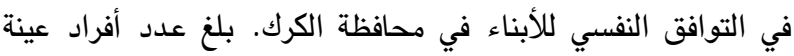

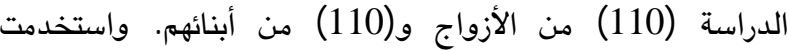
الدراسة مقياس الطلاق العاطفي، ومقياس التوافق النفسي للأبناء.

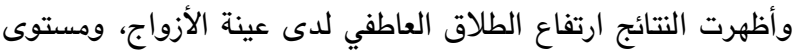

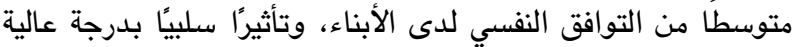
للطلاق العاطفي لدى الزوجين على التوافق النفسي لأبنائهم. من خلال الاطلاع على الدراسات السابقة، يتضح أن الدراسة الحالية ركزت بشكل خاص على تناول متغيرات لم يتم تناولها معًا سابقًا، وهي مواتع التواصل الاجتماعي والصلابة النفسية والطلاق

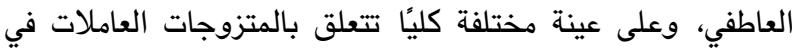

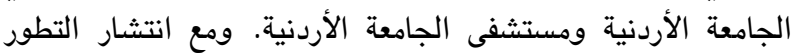

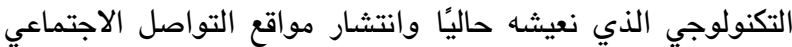

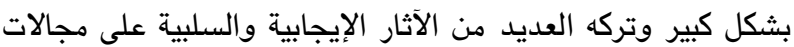

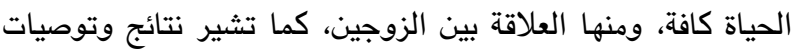

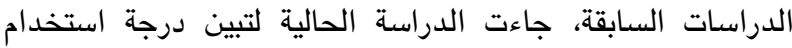

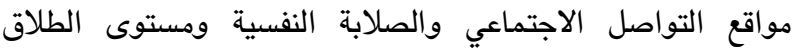

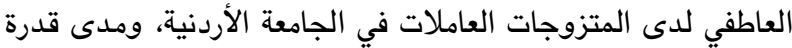

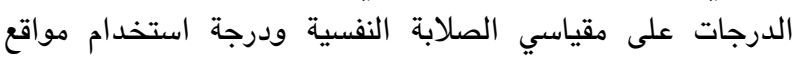
التواصل الاجتماعي على التنبؤ بالطلاق العاطفي.

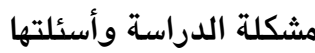

لاحظ الباحثان خلال عملهما في القطاع الجامعي والمدرسي

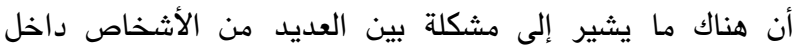

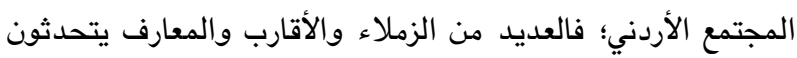

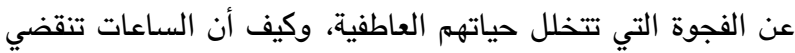

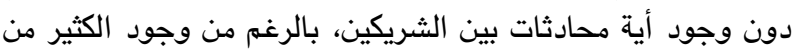

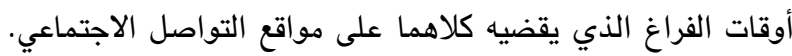
ومع تكرار سماع مثل هذه المواقف من أشخاص مختلفين على الفي التهاصي

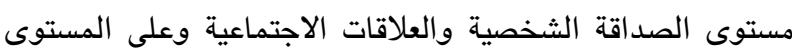

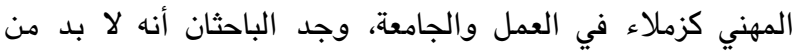

آب وفقًا لبعض المتغيرات مثل: عدد ساعات الاستخدام، والجنس، وكات

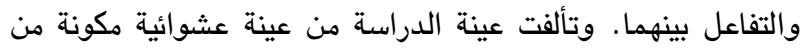
(436) فردًا (309 ذكور و 127 أنثى) من المتزوجين السعوديين،

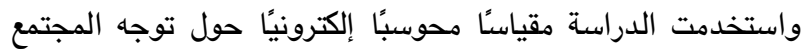

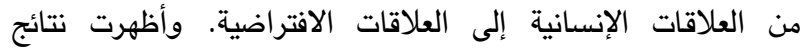
الدراسة وجود أثر دال إحصائيًا لعامل عدد ساعات الإتات استخدام الواتس التسات

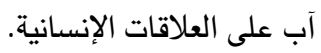

وأجرت السميحيين (Al-Smeheen, 2016) دراسة هدفت إلى التعرف على العلاقة بين التوافق الزواجي والصلابة النفسية

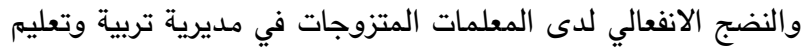

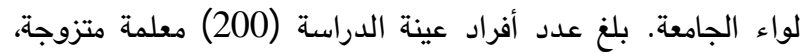

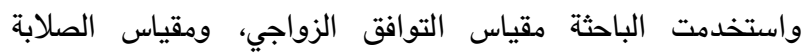

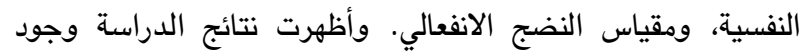

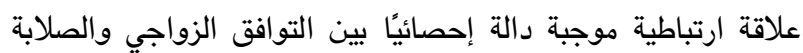

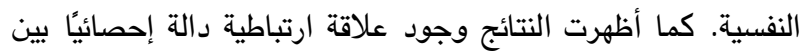
التوافق الزواجي والنضج الانفعالي.

وقام الطنبالي (Al-Tunbali, 2016) بدراسة حول أثر إدمان

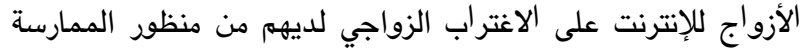

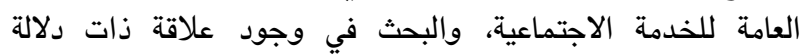
إحصائية بين إدمان الأزواج للإنترنت والاغتراب الزابت الزواجي لديهمه. تألفت

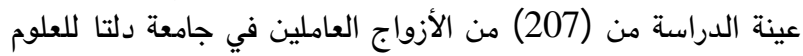

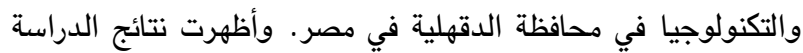
وجود علاقة طردية دالة إحصائيًا بين إدمان الأزواج للإنترنت التاني

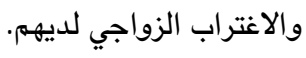

وأجرت لكحال وزايدي (Lkhal \& Zaidi 2017) دراسة هدفت إلى فحص أثر الاستخدام المفرط لمواتع التواصل الاجتماعي

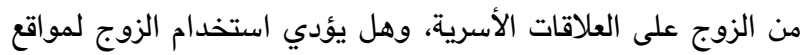

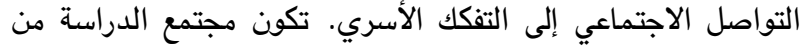

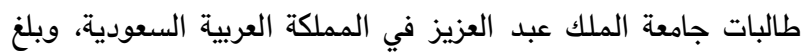
عدد أفراد عينة الدراسة (862) طالبة، وقامت الباحثتان بتطوير

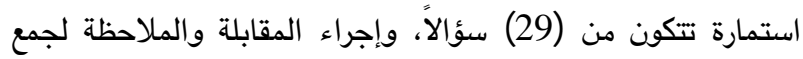

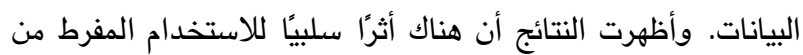
الزوج لمواقع التواصل الإجتماعي على العلاقات الأسرية.

وقام الحمد وآخرون (Al-Hamad et al., 2017) بدراسة هدفت إلى الكثف عن مستوى الصلابة النفسية لدى الطالبات المتزوجات وغير المتزوجات من كلية إربد التابعة لجامعة البلقاء

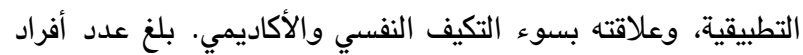
عينة الدراسة (191) طالبة، واستخدم الباحثون مقياس الصلابة

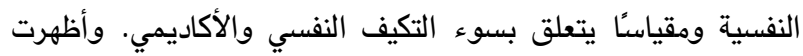
النتائج أن مستوى الصلابة النفسية لدى الطالبات المتزوجات وغيري

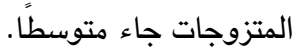


العاملات في الجامعة الأردنية. وتحاول الدراسة الإجابة عن الأسئلة

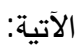

1. ما درجة استخدام المتزوجات العاملات في الجامعة الأردنية

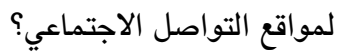

2. ما مستوى الصلابة النفسية لدى المتزوجات العاملات في

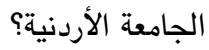

3. ما مستوى الطلاق العاطفي لدى المتزوجات العاملات في

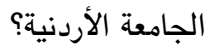

4. ما نسبة التباين الذي يفسره استخدام مواقع التواصل

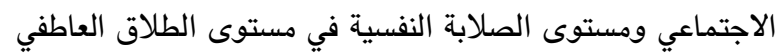

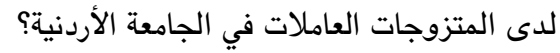

أهداف الدراسة

تهدف الدراسة الحالية إلى التعرف إلى درجة استخدام مواقع

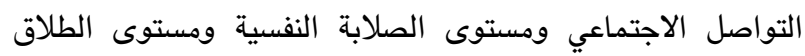

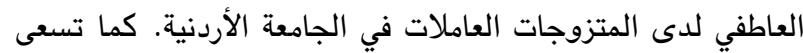

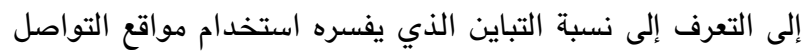

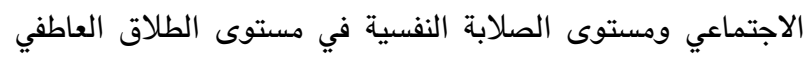
لدى المتزوجات العاملات في الجامعة الأردنية.

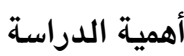

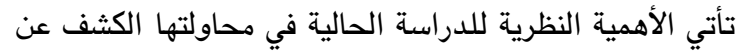

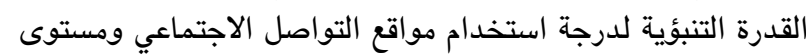

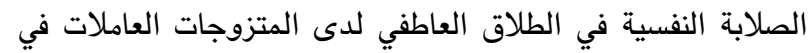

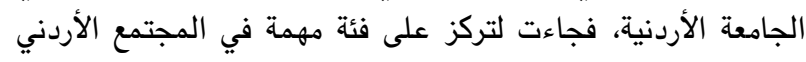

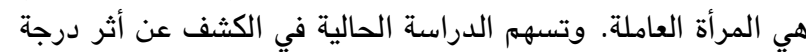

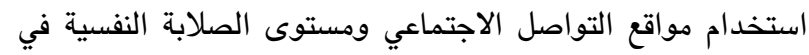

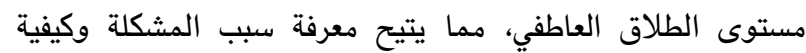

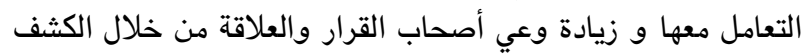

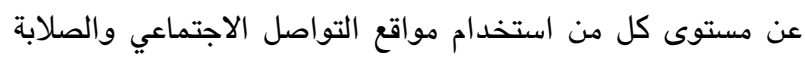

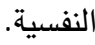

وتقدم نتائج هذه الدراسة بيانات حول طييعة العلاقة بين

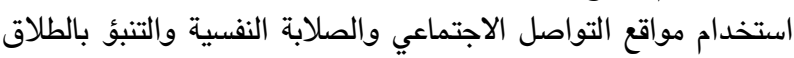

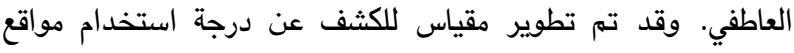
التواصل الاجتماعي، ومقياس للكثف عن عن مستوى الصفي الصلابة النفسية،

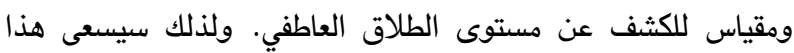

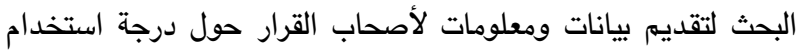

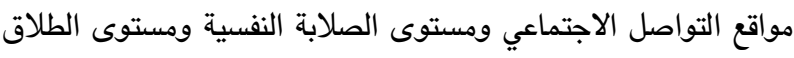

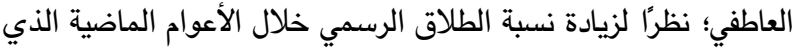

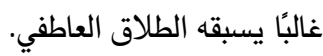

بحث هذه المشكلة والتوسع فيها . ففي الآونة الأخيرة زادت الخلافات

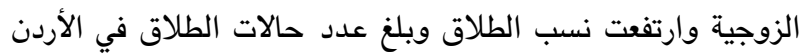
لعام (2017) حسب إحصائيات دائرة قاضي القضاة (21210)

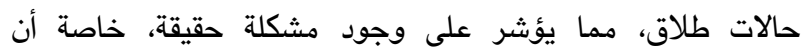

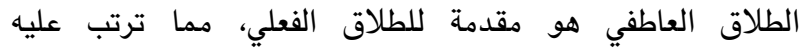
استحداث مكاتب الإصلاح الأسري (Abu Zaid, 2011).

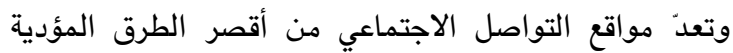

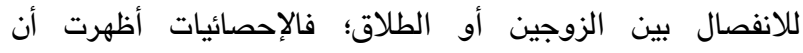

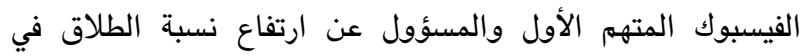

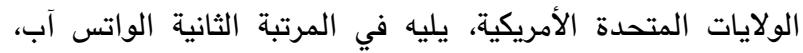

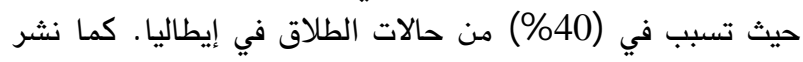
موقع ديفورس (Divorce) أن الفيسبوك تسبب في ثلث الث حالات

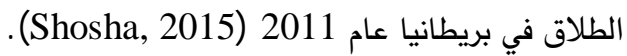
وأجرى الزعبي (Al-Zoubi, 2014) من مديرية الإفتاء في الأردن عام (2014) دراسة لمعرفة أسباب الطلاق. تكونت العينة

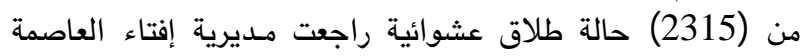

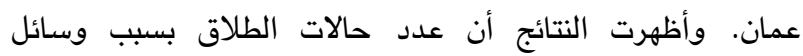

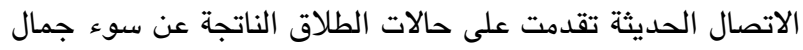

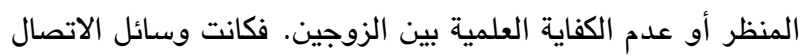

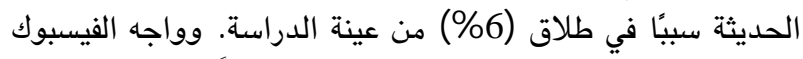

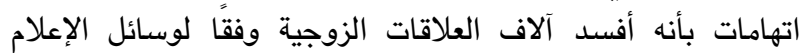

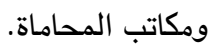

فالاستخدام المفرط للتكنولوجيا ساهم في ترك الأثر الكبير

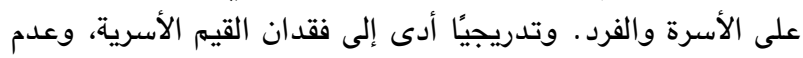

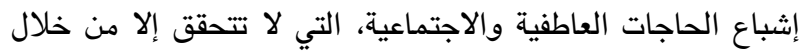

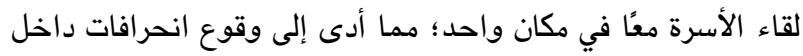

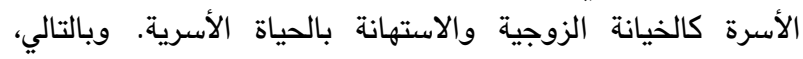

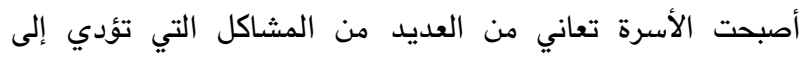

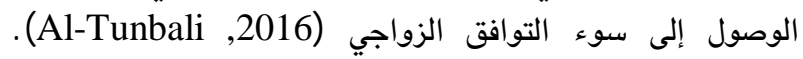

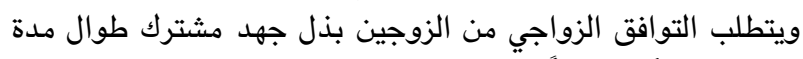

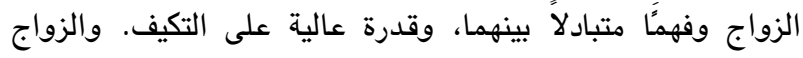

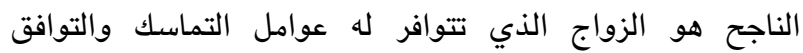
والصلابة النفسية، رغم كل ما يواجه الأزواج من مشكلات وضغوط الزواج

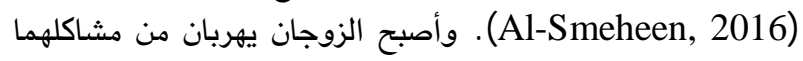

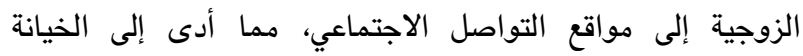

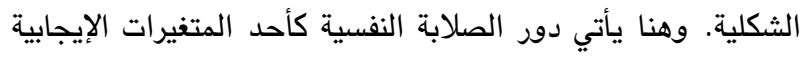

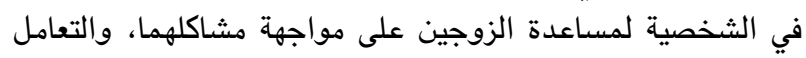

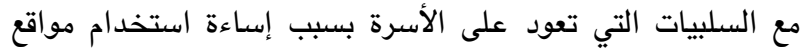

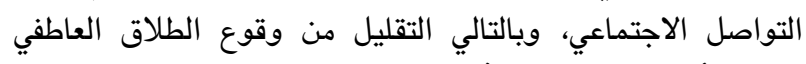

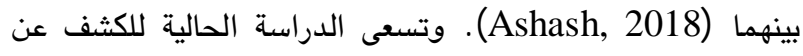

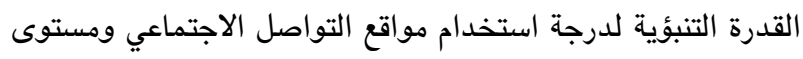
الصلابة النفسية في الطلاق العاطفي لدى لئه عينة من المتزوجات 
منهج الدراسة

اعتمدت الدراسة المنهج الوصفي التحليلي.

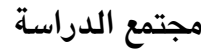

تكون مجتمع الدراسة من المتزوجات العاملات في الجامعة

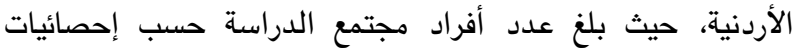

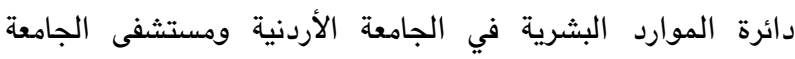

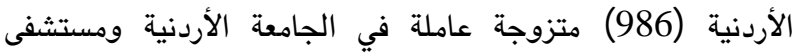

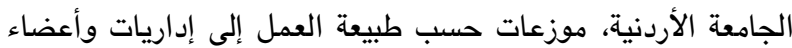

$$
\text { هيئة تدريس. والجدول (1) يوضح ذلك. }
$$

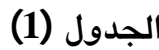

توزيع مجتمع الدراسة وفقاً لطبيعة العمل

\begin{tabular}{|c|c|c|}
\hline النسبة المئوية & العدد & طبيعة العمل \\
\hline$\% 71$ & 697 & إداريات \\
\hline$\% 29$ & 289 & أعضاء هيئة تدريس \\
\hline$\% 100$ & 986 & المجموع \\
\hline
\end{tabular}

عينة الدراسة - م

استخدم أسلوب العينة العشوائية الطبقية، حيث تم تقسيم

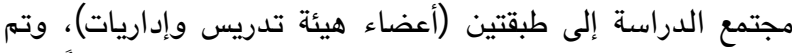

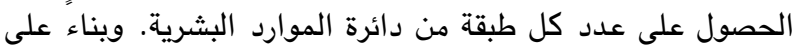
ذلك تم تحديد حجم العينة لكل طبقة من طبقات المجتمع بماء

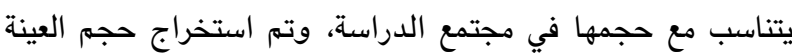

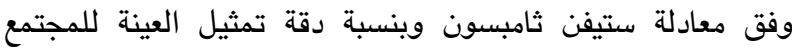
(95\%) عند هامش خطأ (0.05). وبلغ حجم العينة (350) وفينة موظفة. والجدول (2) يوضح توزيع أفراد عينة الدراسة وفقاً لطبيعة

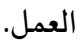

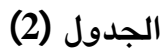

توزيع أفراد عينة الدراسة ونقاً لطبيعة العمل

\begin{tabular}{|c|c|c|}
\hline النسبة المئوية & العدد & طبيعة العمل \\
\hline$\% 71$ & 247 & إداريات \\
\hline$\% 29$ & 103 & أعضاء هيئة تدريس \\
\hline$\% 100$ & 350 & المجموع \\
\hline
\end{tabular}

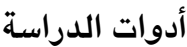

لتحقيق أهداف الدراسة، تم استخدام الأدوات الآتية:

أولاً: مقياس استخدام مواقع التواصل الاجتماعي: بعد الاطلاع

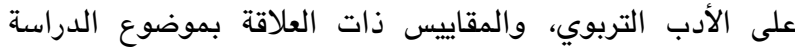
Al-Shamrani, 2015; Al-Shehri, 2013;)

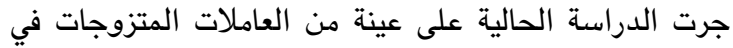

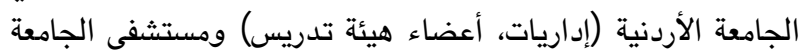

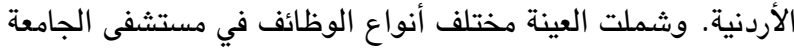

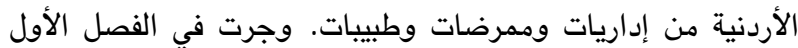
للعام الجامعي 2018 /2019. وتتحدد نتائج الدراسة باستجابات العاملات المتزوجات على

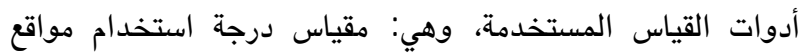
التواصل الاجتماعي، ومقياس الصلابة النفسية، ومقياس الطلاق العاطفي، وما توفر لها من دلالات صدق وثبات.

$$
\text { التعريفات الإجرائية }
$$

• المرأة العاملة المتزوجة: هي المرأة التي تعمل خارج المنزل وتحصل على أجر مادي مقابل عملها، وهي تقوم بدورين أساسيين

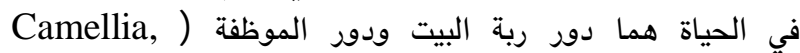

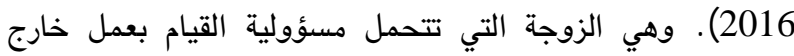

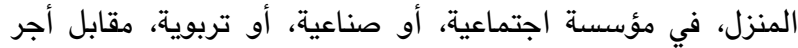

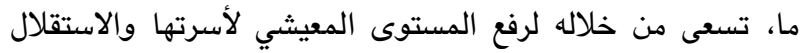

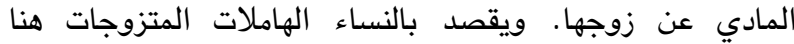
اللواتي أجبن على مقاييس الدراسة.

• استخدام مواقع التواصل الاجتماعي: درجة استخدام مجموعة

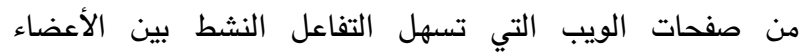

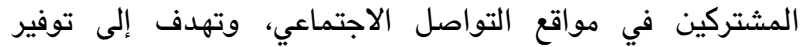
مختلف وسائل الاهتمام التي تساعد الأعضاء على التى التفاعل باعل بين بعضهم البعض (Fahmi, 2018). ويقاس بالدرجة التي حصلت التئ عليها المستجيبة على مقياس درجة استخدام مواقع التواصل الاجتماعي المستخدم في الدراسة. • الصلابة النفسية: مجموعة من السمات تتمثل في اعتقاد لدى الدى العيد الفرد بفاعليته وقدرته على استثمار كل المصادر النفسية والبيئية المتاحة كي يدرك بفاعلية أحداث الحياة الضاغطة الشاقة إدراكًا غير الفير

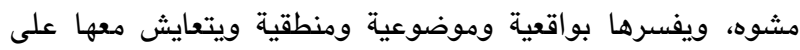

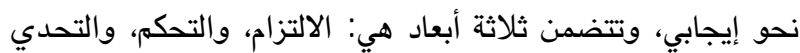

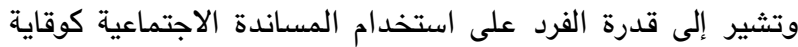

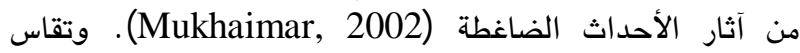
بالدرجة التي حصلت عليها المستجيبة على مقياس الصلابة النفسية

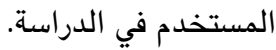
• الطلاق العاطفي: حالة من الانعزال العاطفي بين الزوجين، بحيث

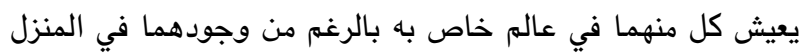

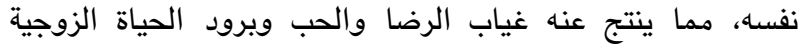
(Hadi, 2012). ويقاس بالدرجة التي حصلت عليها المستجيبة النية على مقياس الطلاق العاطفي المستخدم في الدراسة. 
الاجتماعي على النحو الآتي:1.33 فأقل) مستوى منخفض من (2.67-1.34) مستوى

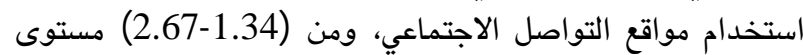
متوسط من استخدام مواقع التواصل الاجتماعي، و (2.68 فأكثر)

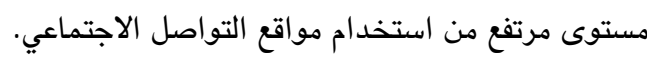

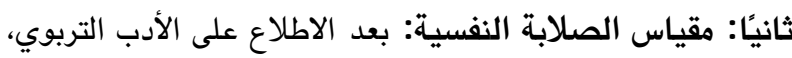

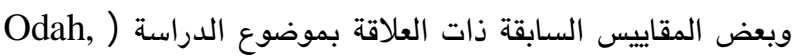
(2010; Al-Ayafi, 2012; Subhi, 2016; المقياس. وتكون المقياس بصورته الأولية من (33) فقرة، وتم تقسيمه إلى ثلاثة أبعاد هي: بعد التحئ بصورته الأولية من والسيطرة، وبعد الالتزام،

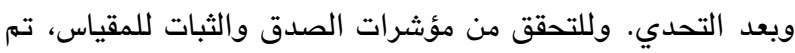
استخراج مؤشرات الصدق والثبات الآتية:

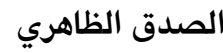

للتحقق من صدق مقياس الصلابة النفسية بصورته الأولية، تم

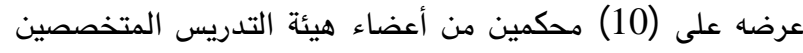

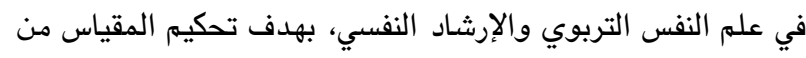

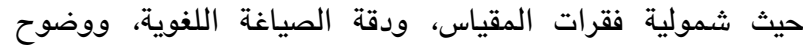

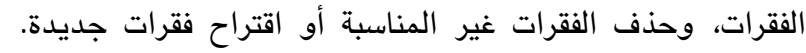

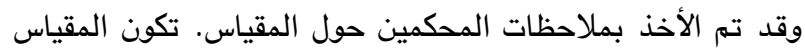

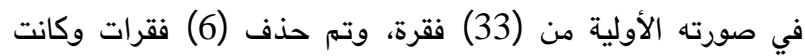

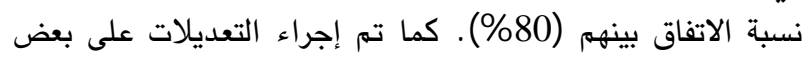
فقرات المقياس بناء على الملاحظات التي قدمها المحكمون.

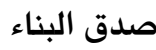

للتحقق من صدق البناء للمقياس، تم تطبيقه على عينة تكونت

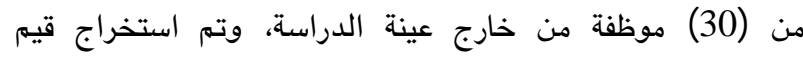

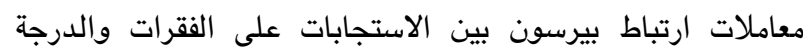

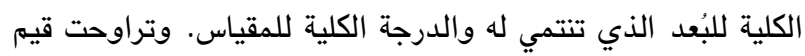

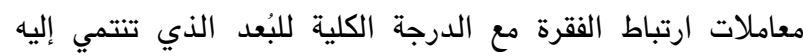

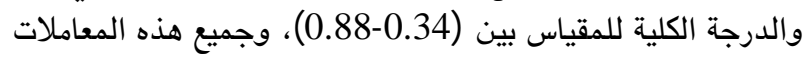

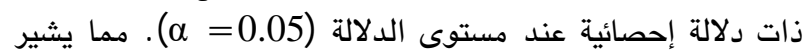

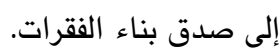

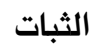

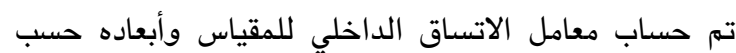

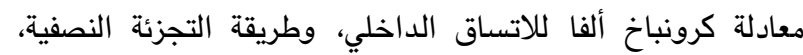

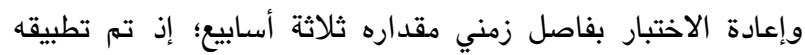

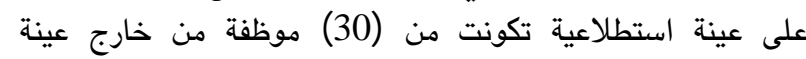

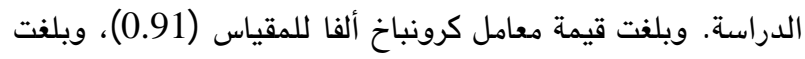

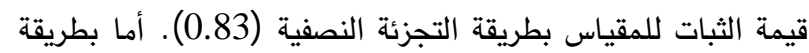

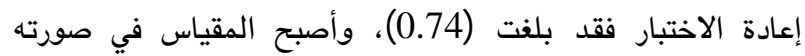
النهائية مكونًا من (27) فقرة الأن
Abdalraheem, 2013 )، تم تطوير مقياس درجة استخدام

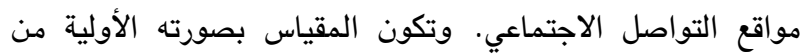

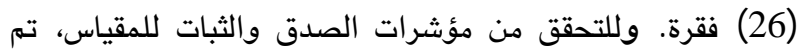

$$
\text { استخراج مؤشرات الصدق والثقات من مؤرات الاتية: }
$$

الصدق الظاهري

للتحقق من صدق مقياس استخدام مواقع التواصل الاجتماعي

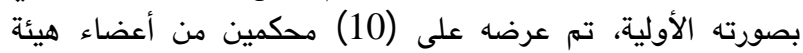

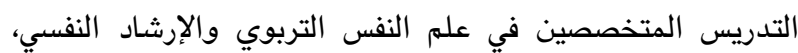

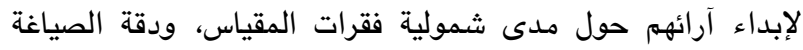

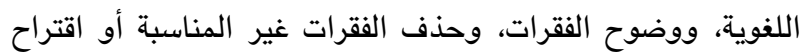

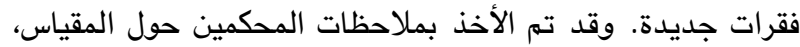

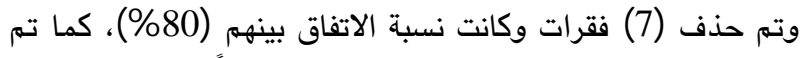

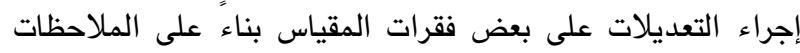
التي قدمها المحكمون.

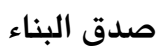
للتحقق من صدق البناء للمقياس، تم تطبيقه على عينة تكونت

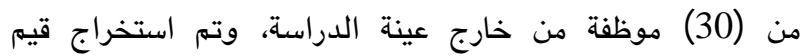

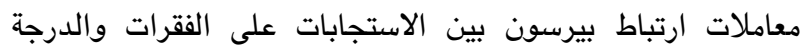

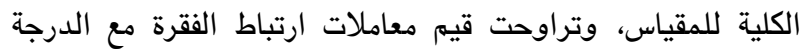

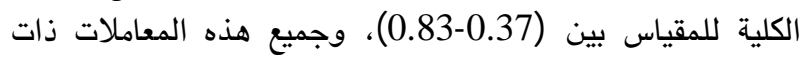

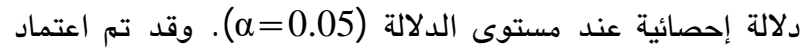

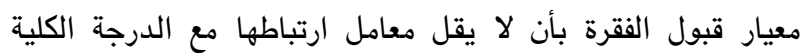

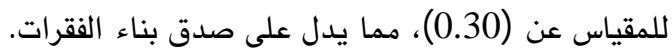
الثبات

تم حساب معامل الاتساق الداخلي للمقياس حسب معادلة

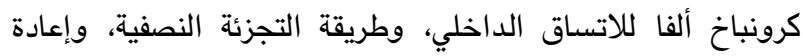

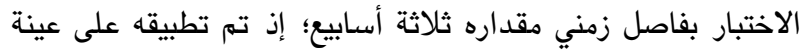

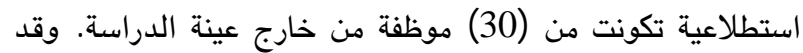

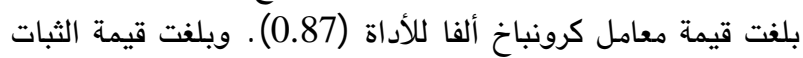

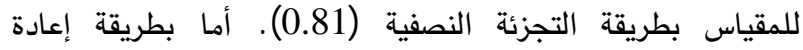
الاختبار فقد بلغت (0.87)، وأصبح المقياس في صورته النهاية

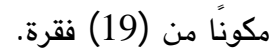
تصحيح المقياس

بهدف تصحيح المقياس، تم اعتماد تدريج ليكرت الخماسي

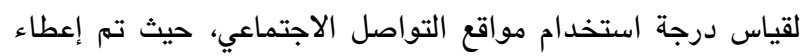

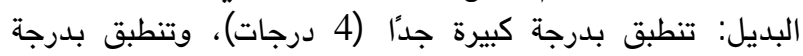

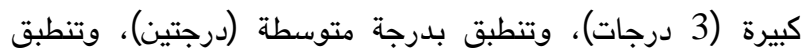

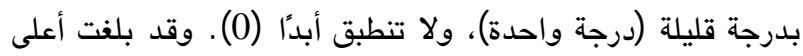

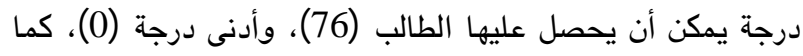

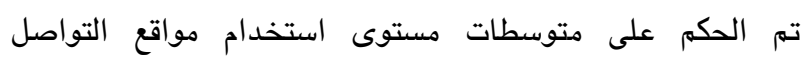




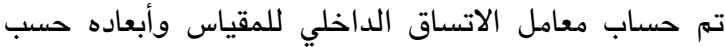

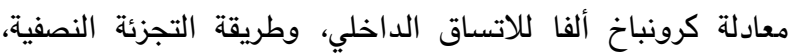

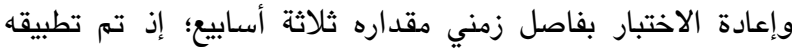

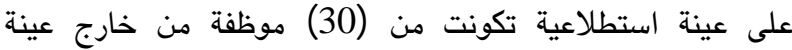

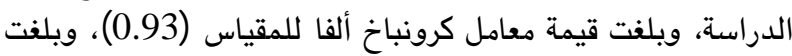
قيمة الثبات للمقياس بطريقة التجزئة النصفية (0.86) . أما بطريقة

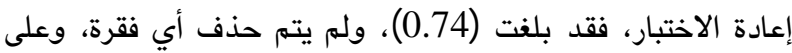
ذلك بقي المقياس في صورته النهاية مكونًا من (33) فقرة.

\section{تصحيح المقياس}

بهدف تصحيح المقياس، تم اعتماد تدريج ليكرت الخماسي

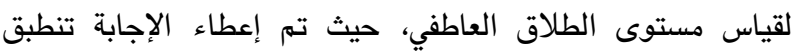
بدرجة كبيرة جدا (5 درجات)، وتنطبق بدرجة كبيرة (4 درجات)،

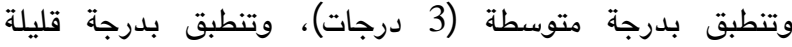

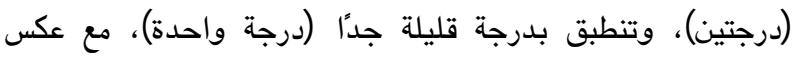

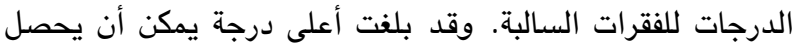

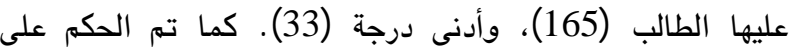
متوسطات تقدير مستوى الطلاق العاطفي على النحو الآتي:2.33) فأقل) مستوى منخفض من الطلاق العاطفي، ومن (3.67-2.34)

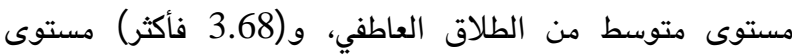

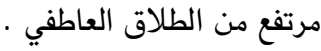

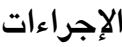

تم تطبيق مقاييس الدراسة الثلاثة على أفراد عينة الدراسة بشكل شخصي، وتوضيح وشرح كيفية تعبئة البيانات المطلوبة

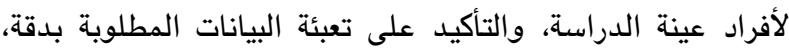
والإجابة عن جميع فقرات المقاييس مع توضيح أن جميع المعلومات سرية، ولن تستخدم إلا لغايات البحث العلمي. وتم توزيع (400)

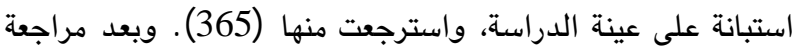

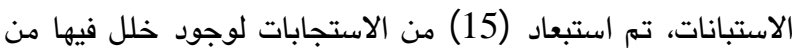
حيث عدم جدية المفحوص و تجاهل عدد من الفقرات.

النتائج

أولاً: النتائج المتعلقة بالسؤال الأول: ما درجة استخدام

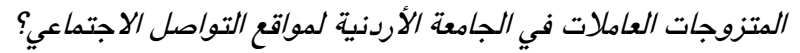
للإجابة عن هذا السؤال، استخرجت المتوسطات الحسابية

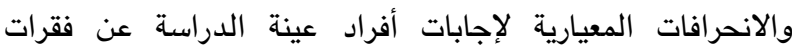
مقياس استخدام مواقع التواصل الاجتماعي والدرجة الكلية، والجدول (3) يوضح ذلك.
بهدف تصحيح المقياس، تم اعتماد تدريج ليكرت الخماسي

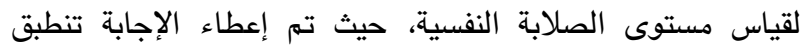
بدرجة كبيرة جدًا (5 درجات)، وتنطبق بدرجة كبيرة (4 درجات)، وتنطبق بدرجة متوسطة (3 درجات)، وتنطبق بدرجة قليلة

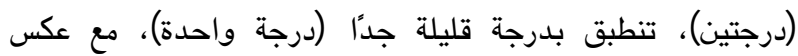

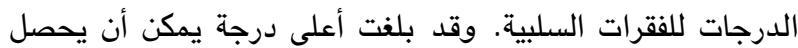

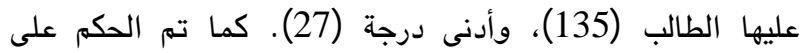

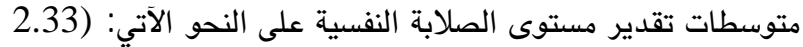
فأقل) مستوى منخفض من الصلابة النفسية، من (3.67-2.34)

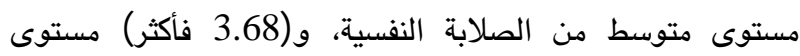

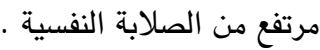

ثالثًا: مقياس الطلاق العاطفي: بعد الاطلاع على الأدب التربوي

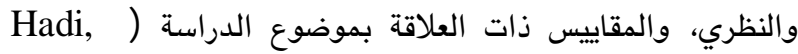
2012; Abdullrahman, 2017; Al-Fatlawi and Jaber, (2012; Mansour, 2009 من (33) فقرة، وقستم إلى ثلاثة أبعاد هي: البعد العاطفي، ويُعد التواصل، والبُعد الفكري. وللتحقق من مؤشرات الصدق والثى لثقات الثبات للمقياس، تم استخراج مؤشرات الصدق والثدي، ولثديات الآتية: الصدق الظاهري

للتحقق من صدق مقياس الطلاق العاطفي بصورته الأولية، تم

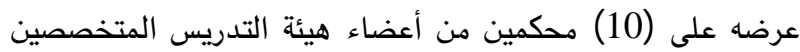

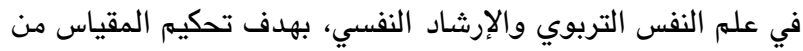
حيث؛ مدى شمولية فقرات المقياس، ودقة الصياغة اللفوية، ووضوح الفقرات، وحذف الفقرات غير المناسبة أو اقتراح فقرات جديدة. وقد تم الأخذ بملاحظات المحكمين حول المقياس. وتكون المقياس في صورته الأولية من (33) فقرة، ولم يتم حذف أي فقرة،

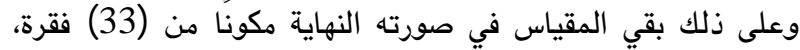

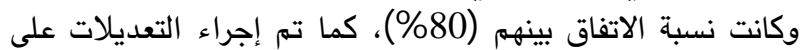

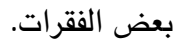

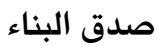

للتحقق من صدق البناء للمقياس، تم تطبيقه على عينة استطلاعية تكونت من (30) موظفة من خارج عينة الدراسة، وتم

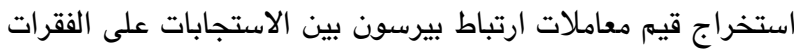

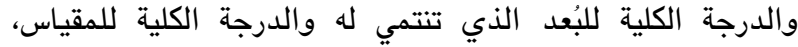

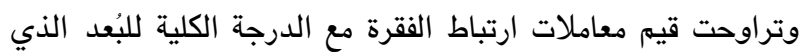

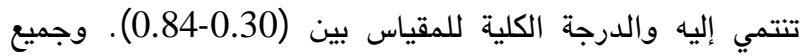
هذه المعاملات ذات دلالة إحصائية عند مستوى الدلالة (مل0. ه)، مما يشير إلى صدق بناء الفقرات. 


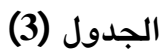

المتوسطات الحسابية والانحرافات المعيارية لإجابات أفراد عينة الدراسة عن نقرات مقياس استخدام مواقع التواصل الاجتماعي والدرجة الكلية مرتبة

تنازلياً

\begin{tabular}{|c|c|c|c|c|c|}
\hline الاستخدام & الالمعياري & الحسابي & 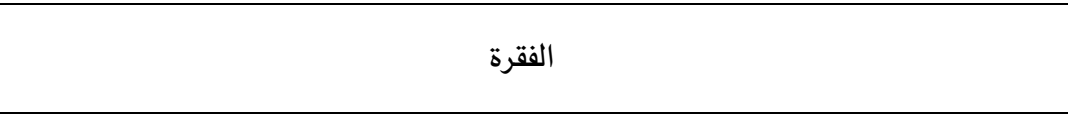 & 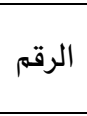 & الرتبة \\
\hline مرتفعة & 0.75 & 3.53 & أستخدم مواقع التواصل الاجتماعي أكثر من أربع ساعات يوميًا & 7 & 1 \\
\hline مرتفعة & 1.08 & 3.39 & أقضي ساعات طويلة في استخدام" مواقع التواصل الاجتماعي دون الإحساس بالوقت & 9 & 2 \\
\hline مرتفعة & 1.00 & 3.38 & أتصفح مواقع التواصل الاجتماعي في المنزل & 6 & 3 \\
\hline مرتفعة & 1.11 & 3.38 & أضيف يوميًا أصدقاء جددًا إلى حساباتي على مواقع التواصل الاجتماعي & 11 & 4 \\
\hline مرتفعة & 1.03 & 3.31 & أقضي وقتا أكثر على مواقع التواصل الاجتماعي من الوقت الذي أقضيه مع أسرتي & 2 & 5 \\
\hline مرتفعة & 1.08 & 3.30 & أقوم بمشاهدة وقراءة حالات الآخرين على مواقع التواصل الاجتماعي & 16 & 6 \\
\hline مرتفعة & 1.05 & 3.29 & أتابع المستجدات الإخبارية من خلال مواقع التواصل الاجتماعي & 19 & 7 \\
\hline مرتفعة & 0.86 & 3.28 & أجد صعوبة في التوقف عن استخدام مواقع التواصل الاجتماعي حتى لو أصابني النعاس & 1 & 8 \\
\hline مرتفعة & 1.21 & 3.26 & استخدامي لمواقع التواصل الاجتماعي سبب لي أضرارًا جسدية & 10 & 9 \\
\hline مرتفعة & 1.21 & 3.19 & بالفشل لحاولي في التقليل من الوقت الذي أقضيه في استخدام مواقع التواصل الاجتماعي باءت & 15 & 10 \\
\hline مرتفعة & 1.23 & 3.07 & أتفاعل بالتعليق على منشورات الآخرين & 17 & 11 \\
\hline مرتفعة & 1.24 & 3.05 & ذهني منشغل بكيفية البقاء متصلة على مواقع التواصل الاجتماعي & 18 & 12 \\
\hline مرتفعة & 1.40 & 2.98 & ينتقد الآخرون كثرة استخدامي لمواقع التواصل الاجتماعي & 14 & 13 \\
\hline 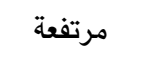 & 1.36 & 2.92 & أتابع حسابات المشاهير & 12 & 14 \\
\hline مرتفعة & 1.39 & 2.91 & أهملت أنشطة حياتي المختلفة بسب انشغالي باستخدام مواقع التواصل الاجتماعي & 8 & 15 \\
\hline مرتفعة & 1.47 & 2.73 & أتواصل مع أصدقائي عبر مواقع التواصل الاجتماعي & 13 & 16 \\
\hline 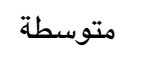 & 1.49 & 2.57 & أتصفح مواقع التواصل الاجتماعي خلال ساعات العمل & 5 & 17 \\
\hline 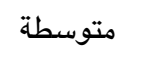 & 1.52 & 2.43 & أشارك الصور أو الحالات على حساباتي على مواقع التواصل الاجتماعي & 4 & 18 \\
\hline 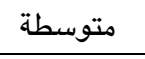 & 1.50 & 2.42 & ينشغل ذهني بسبب انقطاع الإنترنت أو عندما أصبح خارج التغطية & 3 & 19 \\
\hline 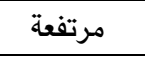 & 0.61 & 3.07 & اقع التواصل الاجتماعي ككل & خدل & كياس \\
\hline
\end{tabular}

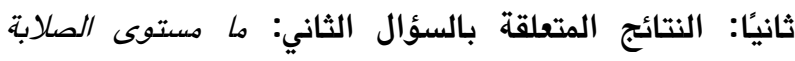

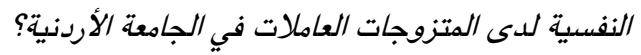

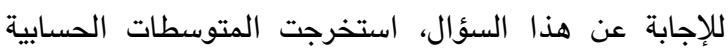

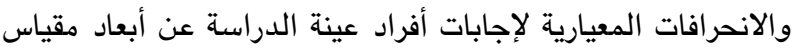

الصلابة والمقياس ككل، والجدول (4) يوضح ذلك.

يظهر من الجدول (3) أن درجة استخدام المتزوجات العاملات

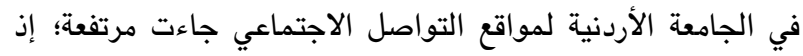

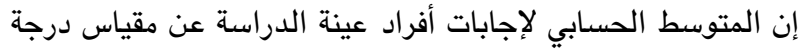

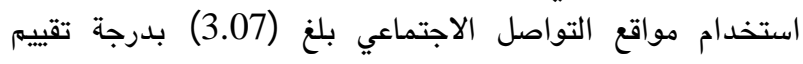

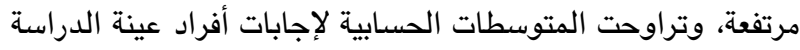
عن فقرات مقياس استخدام التواصل الاجتماعي بين (2.42-

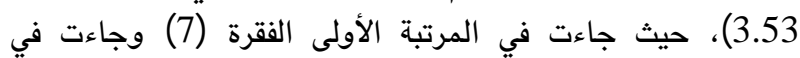

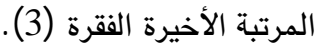

(4) الجدول

المتوسطات الحسابية والانحرافات المعيارية لإجابات أفراد عينة الدراسة عن أبعاد مقياس الصلابة النفسية والمقياس ككل مرتبة تنازليا

\begin{tabular}{|c|c|c|c|c|c|}
\hline المستوى & الانحراف المعياري & المتوسط الحسابي & البُعد & الرقم & الرتبة \\
\hline متوسط & 0.84 & 3.04 & بعد التحكم والسيطرة & 1 & 1 \\
\hline متوسط & 0.68 & 2.76 & بعد التحدي & 3 & 2 \\
\hline متوسط & 0.83 & 2.74 & بعد الالتزام & 2 & 3 \\
\hline متوسط & 0.52 & 2.84 & مقياس الصلابة النفسية ككل & & \\
\hline
\end{tabular}


حسابي (2.76)، واحتل المرتبة الثالثة بُعد الالتزام بمتوسط

حسابي (2.74).

ثالثًا: النتائج المتعلقة بالسؤال الثالث: ما مستوى الطلاق العاطفي لدى المتزوجات العاملات في الجامعة الأردنية؟ بالفوال

للإجابة عن هذا السؤال، استخرجت المتوسطات الحسابية

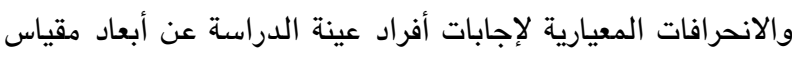
الطلاق العاطفي والمقياس ككل، والجدول (5) يوضح ذلك.
يظهر من الجدول (4) أن مستوى الصلابة النفسية لدى

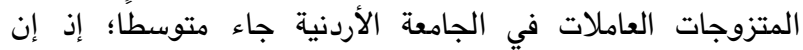

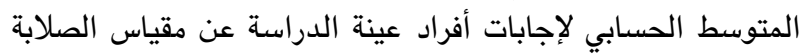

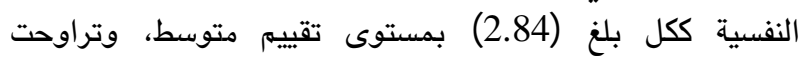
المتوسطات الحسابية لإجابات أفراد عينة الدراسة عن ألفئ أبعاد

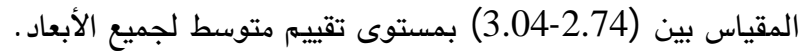

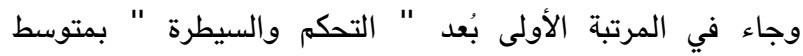

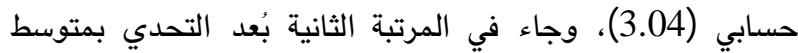

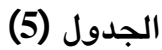

المتوسطات الحسابية والاندرافات المعيارية لإجابات أفراد عينة الدراسة عن أبعاد مقياس الطلاق العاطفي والمقياس ككل مرتبة تنازليا

\begin{tabular}{|c|c|c|c|c|c|}
\hline المستوى & الانحراف المعياري & المتوسط الحسابي & البُعد & الرقم & الرتبة \\
\hline متوسط & 0.88 & 3.17 & بُعد التواصل & 2 & 1 \\
\hline متوسط & 0.74 & 3.04 & البُعد العاطفي & 1 & 2 \\
\hline متوسط & 0.84 & 2.79 & البعد الفكري & 3 & 3 \\
\hline متوسط & 0.75 & 3.00 & لملاق العاطفي ككر & & \\
\hline
\end{tabular}

رابعًا: النتائج المتعلقة بالسؤال الرابع: ما نسبة مستوى التباين

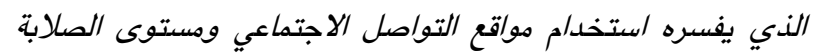

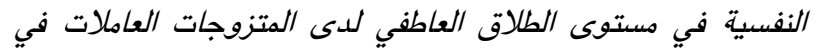

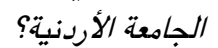

للإجابة عن هذا السؤال، حسبت قيم معاملات الارتباط الخطية

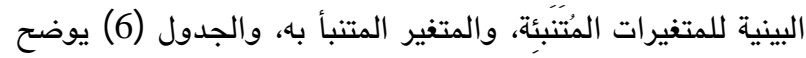
زلك.

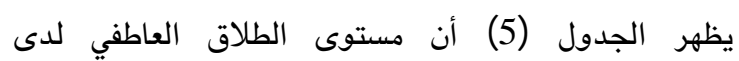

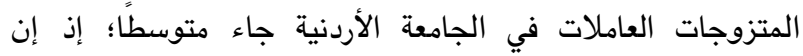

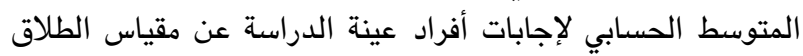

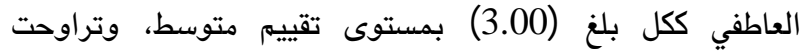

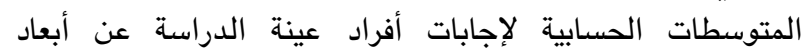

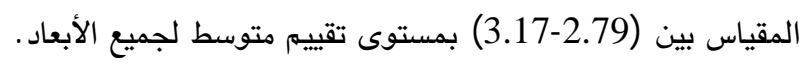

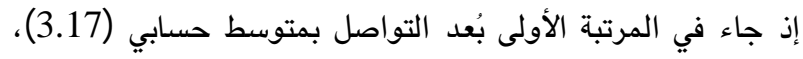

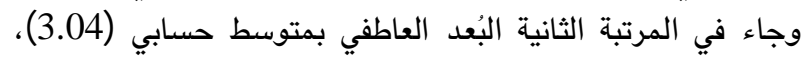

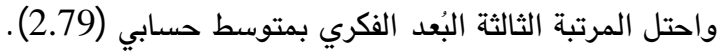

(6) - الجدول

معاملات الارتباط البينية للمتغير المُتَبَاً به والمتغيرات المُتَبَبَّة للعينة الكلية

\begin{tabular}{|c|c|c|}
\hline الطلاق العاطفي & & 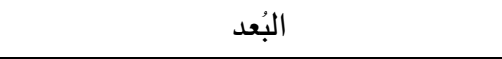 \\
\hline $0.74 *$ & معامل الارتباط & \multirow{2}{*}{ درجة استخدام مواقع التواصل الاجتماعي } \\
\hline 0.00 & الدلالة الإحصائية & \\
\hline $0.60 *$ & معامل الارتباط & \multirow{2}{*}{ مستوى الصلابة النفسية } \\
\hline 0.00 & الدلالة الإحصائية & \\
\hline
\end{tabular}

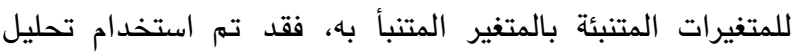

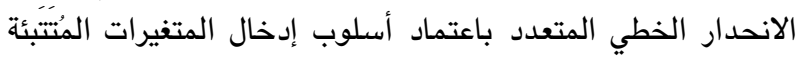

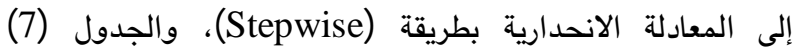
يوضح ذلك.
يلاحظ من الجدول (6) وجود علاقة ارتباطية طردية ودالة

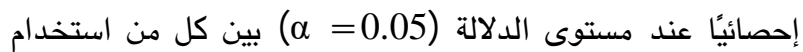

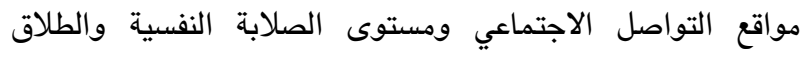

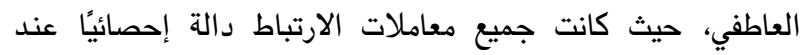
مستوى الدلالة (0.05 = a) . وبهدف الكثف عن القدرة التنبؤية 
نتائج اختبار الفرضيات الانحدارية للمتغير المتنبأ ومعامل الارتباط المتعدد له ومقدار تفسيره حسب أسلوب (Stepwise) المتغيرات المُتَبئة على المعادلة الانحدارية للعينة الكلية

\begin{tabular}{|c|c|c|c|c|c|c|c|c|c|}
\hline \multicolumn{4}{|c|}{ إحصائيات التغير } & \multirow[b]{2}{*}{ للتقدير الخطأ } & \multirow[b]{2}{*}{ المعدل } & \multirow[b]{2}{*}{2} & \multirow[b]{2}{*}{ J } & \multirow[b]{2}{*}{ 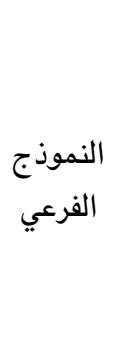 } & \multirow[b]{2}{*}{ المتتبأ به } \\
\hline 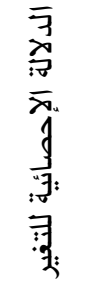 & 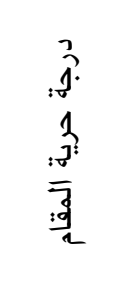 & $\begin{array}{l}3 \\
3 \\
\frac{3}{3} \\
\frac{3}{3}\end{array}$ & 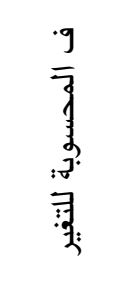 & & & & & & \\
\hline 0.00 & 348.00 & 1.00 & 416.04 & 0.50 & 0.54 & 0.54 & 0.74 & 1 & الطلاق \\
\hline 0.00 & 347.00 & 2.00 & 311.41 & 0.45 & 0.64 & 0.64 & 0.80 & -2 & العاطفي \\
\hline \multicolumn{10}{|c|}{ أ المتنبئات: (ثابت الانحدار)؛ استخدام مواقع التواصل الاجتماعي } \\
\hline
\end{tabular}

أصدقاء جدد والاطلاع على آخر المستجدات الدولية والمحلية

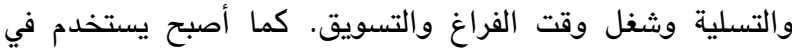

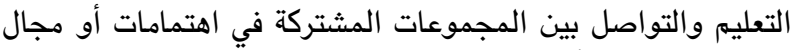

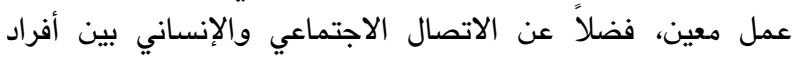

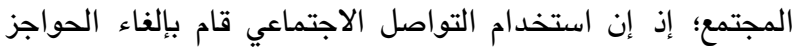

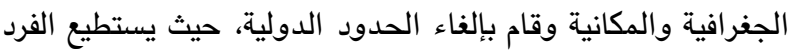
التواصل مع الآخرين ببساطة وسهولة بغض النفاء النظر عن البعد الدافية الجغرافي والمكاني.

كما يمكن تفسير هذه النتيجة بأن مواقع التواصل الاجتماعي

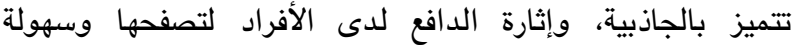

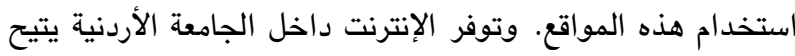

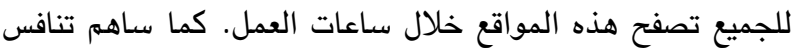

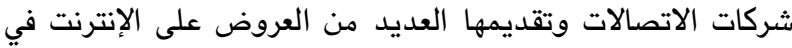

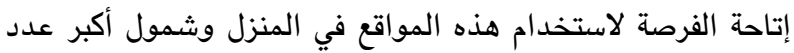

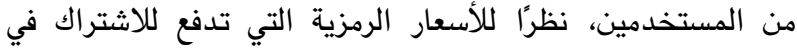

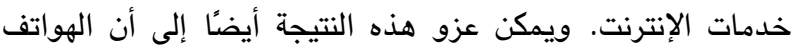

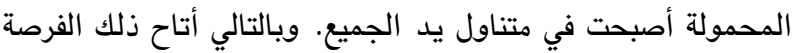
للمستخدمين لتصفح هذه المواقع في أي زمان مكان، وليس حكرًا

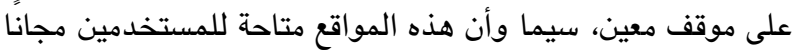
دون أي رسوم أو تكلفة إضافية، يضاف إلى ذلك طوان الكول ساعاعات

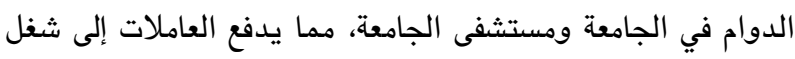

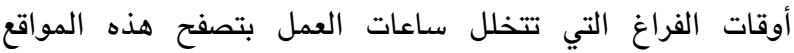
المختلفة و وتتفق هذه النتيجة مع نتائج العديد من الدراسات السابقة العمات Lkhal abd Al-Tubali, 2019; Al-Tunbali, 2019; ) (Zaidi, 2017 ومواقع التواصل الإجتماعي، وعلاقة طردية بين إدمان الأزواج
يتضح من الجدول (7) أن النموذج التنبؤي الخاص

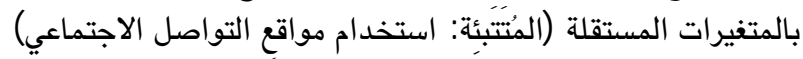

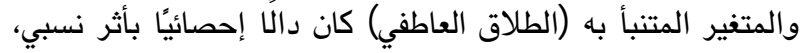

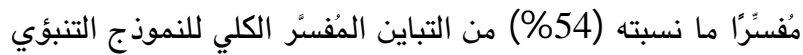

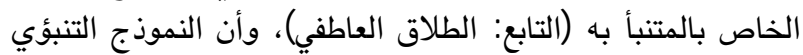

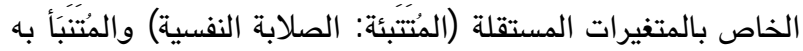

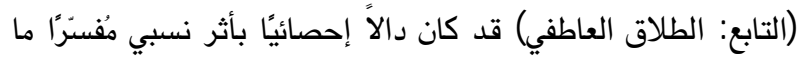

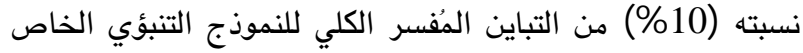

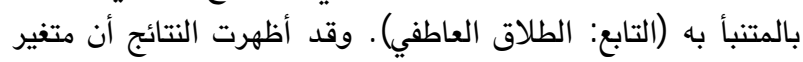

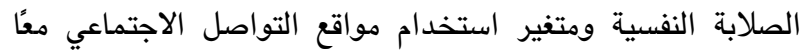

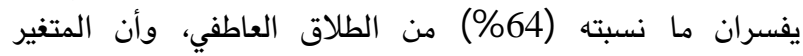

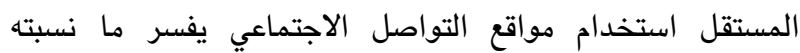

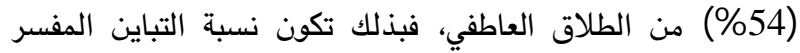

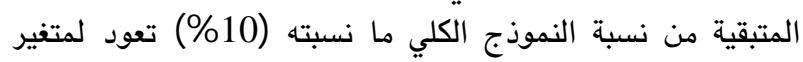

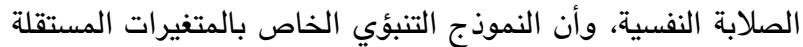

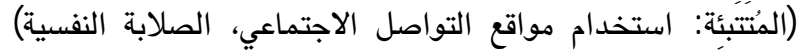

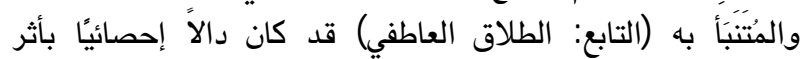

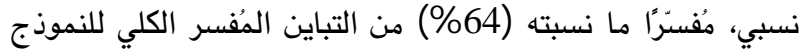
التنبؤي الخاص بالمتنباً به (التابع: الطلاق العاطفي).

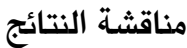
أولاً: مناقشة النتائج المتعلقة بالسؤال الأول: جاءت درجة

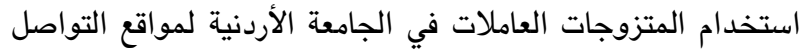

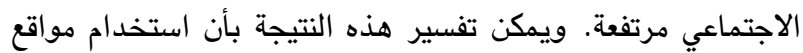

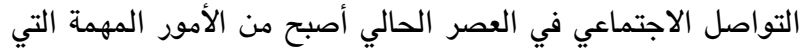

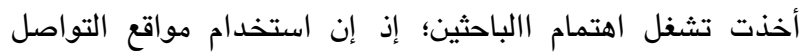

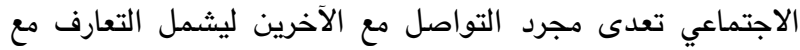


فرضت على المرأة العديد من القيود، إلا أنها تحاول جاهدة التعامل

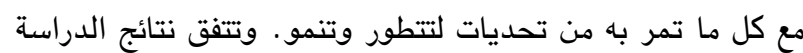

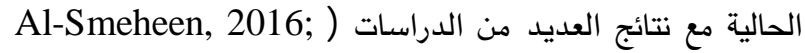

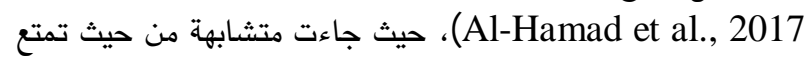

المتزوجات بدرجة متوسطة من الصلابة النفسية.

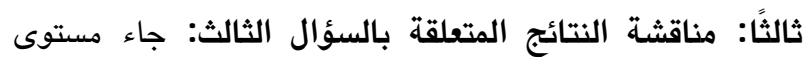
الطلاق العاطفي لدى المتزوجات العاملات في الجامعة الأردنية

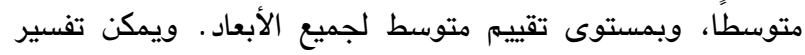

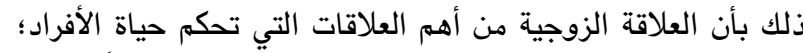

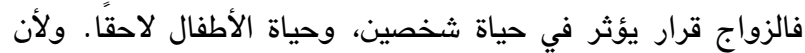

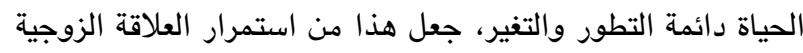

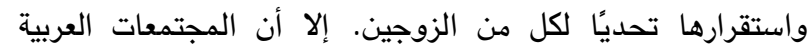

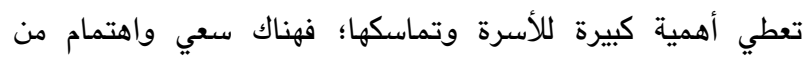
الطرفين للحفاظ على الأسرة من الضياع واحتضان أطفالهما .

كما أظهرت النتائج المتعلقة بالبعد العاطفي أن المستوى العام

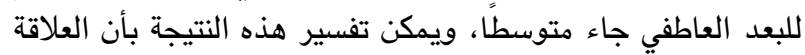

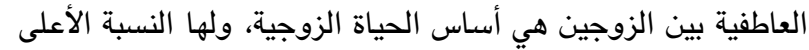

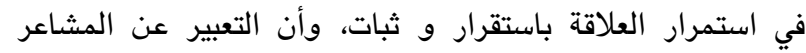

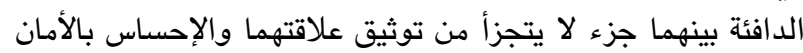

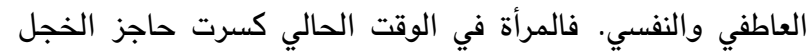

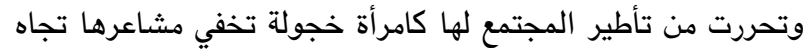

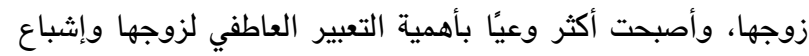

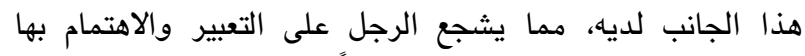

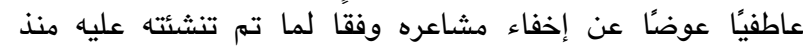

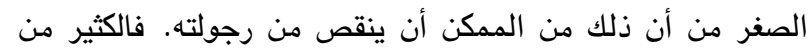

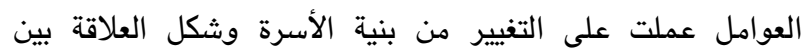

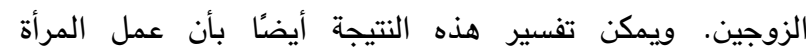

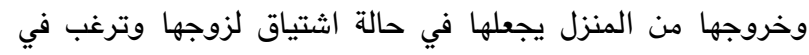

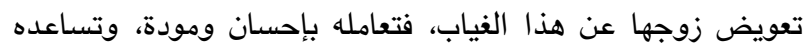

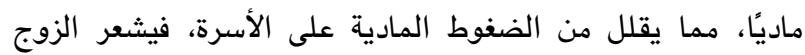
بأهمية زوجته ودورها الفعال، مما يزيد العاطفة بينهما. وفيما يتعلق ببعد التواصل، فقد أظهرت النتائج أن المستوى لئواء

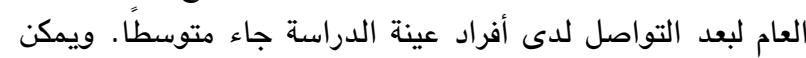

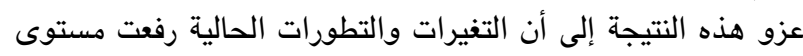

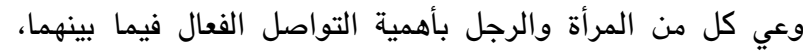

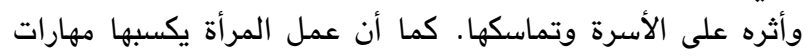

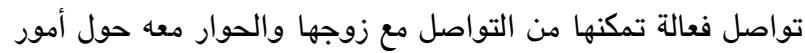

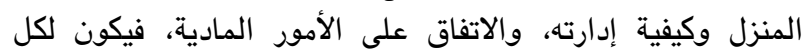

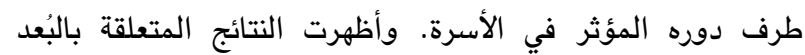

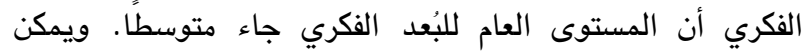

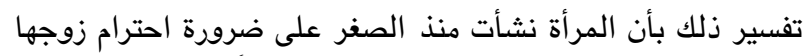
ورأيه وعاداته وتقاليده، مما يجعلها أكثر تقبلًا للاختلافات بـات بينها

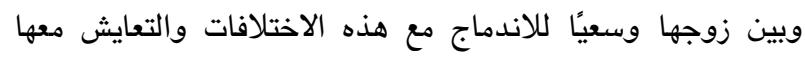

للإنترنت والاغتراب الزواجي، ووجود أثر سلبي للاستخدام المفرط من قبل الزوج على العلاقات الأسرية والتفكك الأسري.

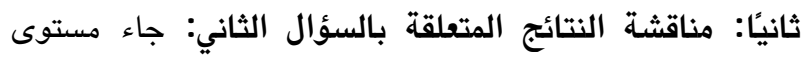
الصلابة النفسية لدى المتزوجات العاملات في الجامعة الأردنية الأنياء

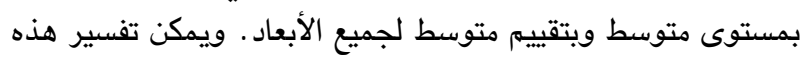

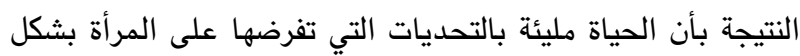

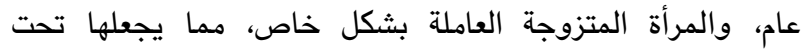

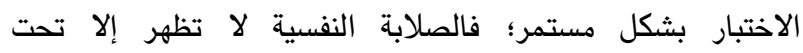

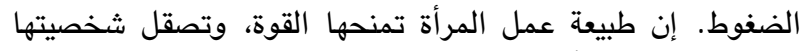

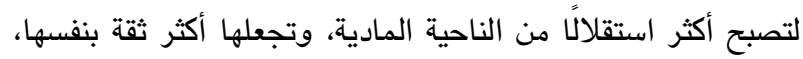

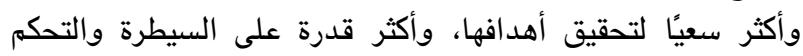

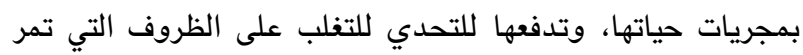

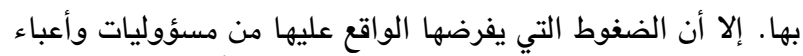

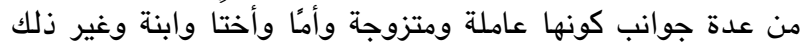

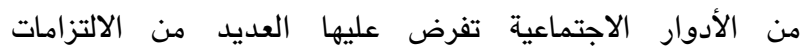

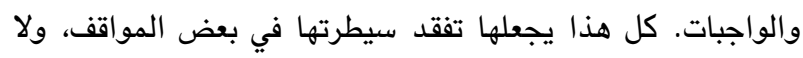

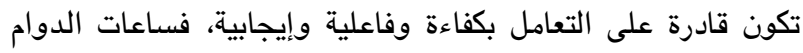
طويلة مع تعدد المسؤوليات المطلوبة منها .

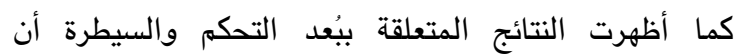

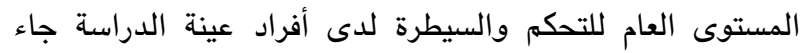

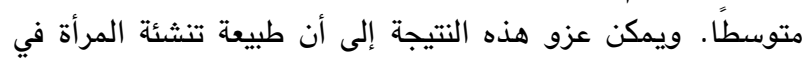

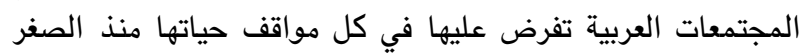

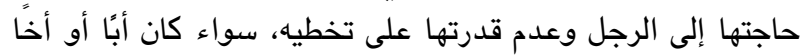

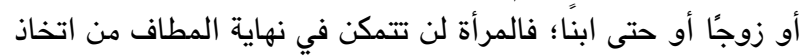

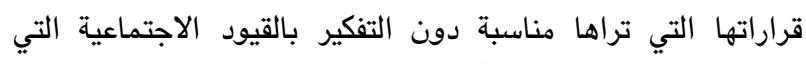

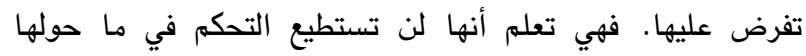
وتحمل المسؤولية الثخصية عن قراراتها.

وفيما يتعلق بيُعد الالتزام، أظهرت النتائج أن المستوى العام

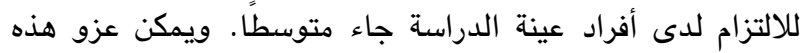

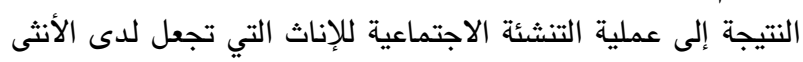

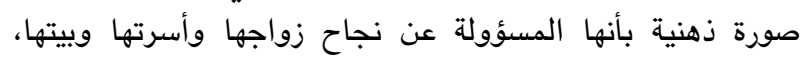

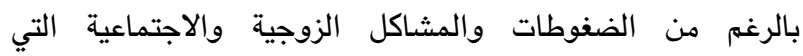

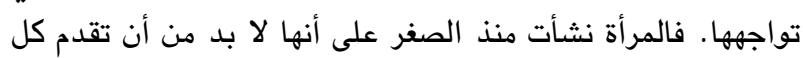

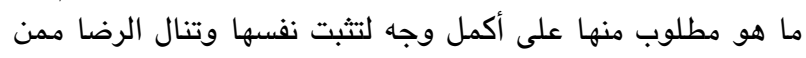
حولها، لكن كثرة المسؤوليات والمتطلبات من المرأة المتزوجة المات ونات

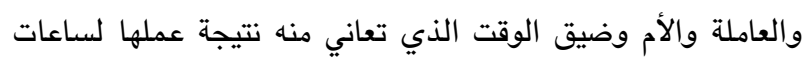

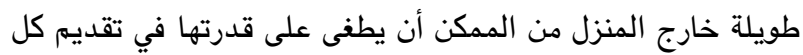

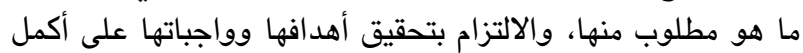

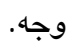

وأظهرت النتائج المتعلقة ببُعد التحدي أن المستوى العام

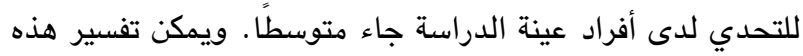
النتيجة بالتحديات التي تفرضها الحياة والتنشئة الاجتماعية التي هئي 
المفروض عليها . فخلال تنشئته منذ الصفر، يتعلم الرجل أنه مصدر

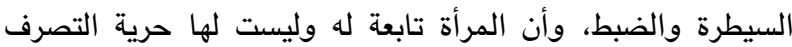

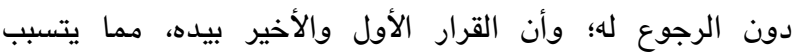

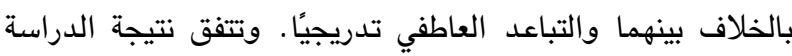

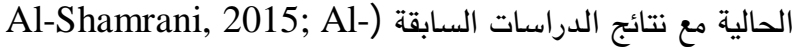
Tunbali, 2016; Lkhal and Zaidi, 2017; Clayton et من حيث وجود علاقة طردية بين إدمان الأزواج

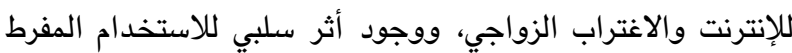

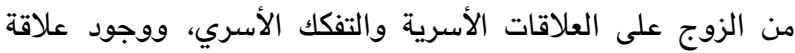

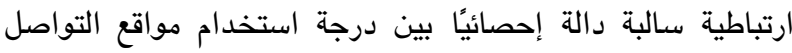

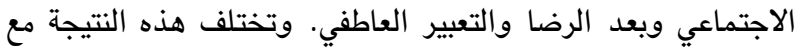

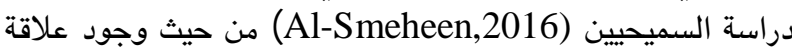
ارتباطية إيجابية بين التوافق الزواجي والصلابة النفسية.

التوصيات

تقدم الدراسة الحالية في ضوء النتائج التوصيات الآتية:

1. إعداد برامج تدرييية لرفع الوعي لدى العاملات المتزوجات في

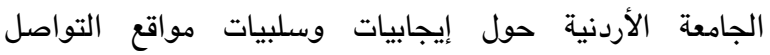
الاجتماعي وكيفية استخدامها وأثرها على الأسرة.

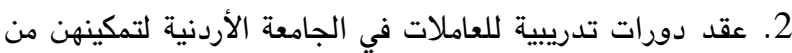

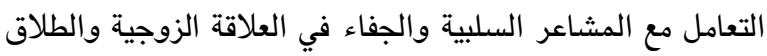

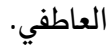

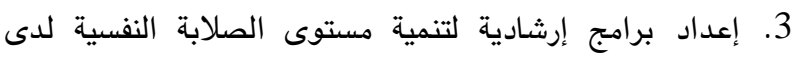
العاملات في الجامعة الأردنية.

4. إعادة تطبيق الدراسة الحالية على عينات وبيئات مختلفة من

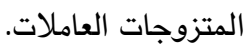

والإبقاء على الحياة الأسرية، مما يساعد على الانسجام الفكري

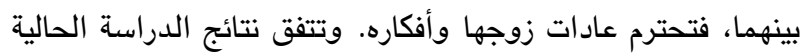

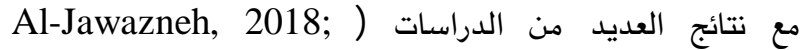
من حيث كون ظاهرة الطلاق العاطفي موجودة (Najdawi, 2018

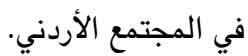

رابعًا: مناقشة النتائج المتعلقة بالسؤال الرابع: أظهرت النتائج أن

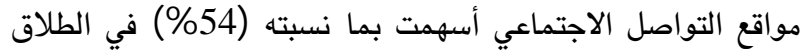

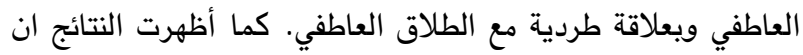

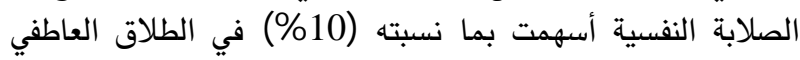

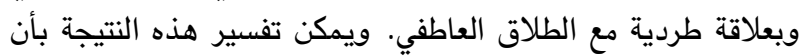

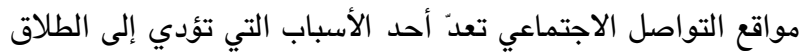

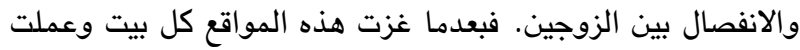

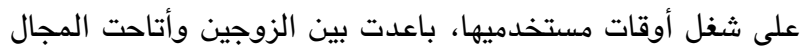

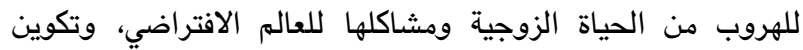

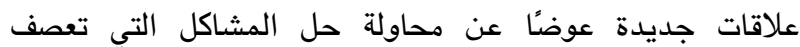

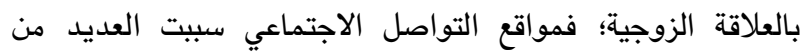

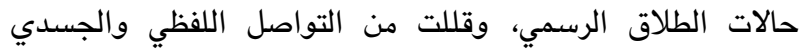

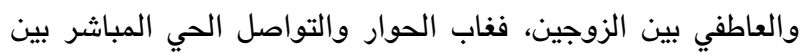

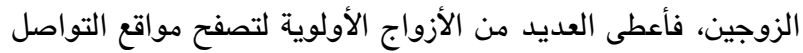

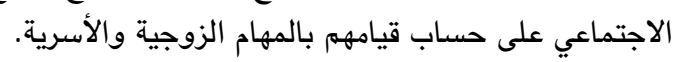
وأظهرت نتائج الدراسة أن الصلابة النفسية تفسر ما نسبته

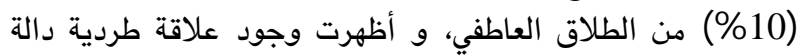

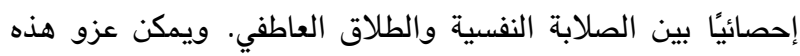

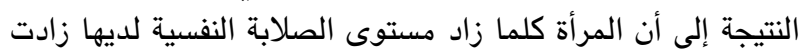

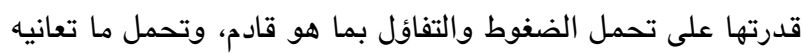

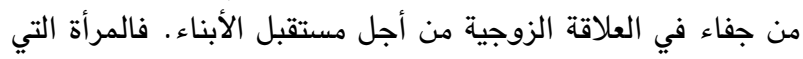

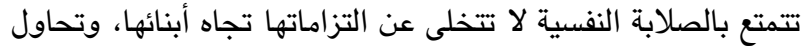

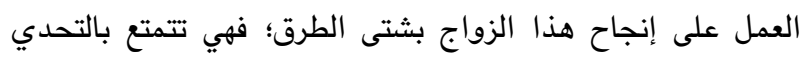
والقدرة على التعامل بإيجابية مع الضفوط. كما يمكن تفسير هذه التهاج

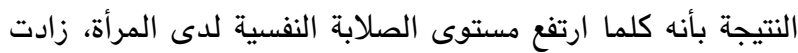

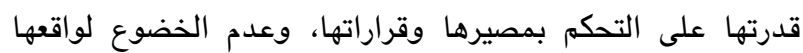

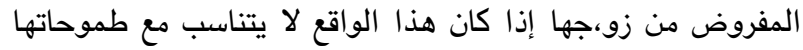

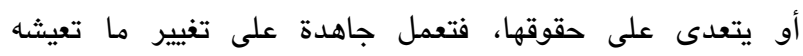

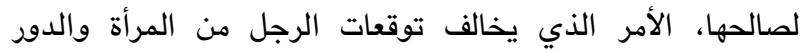




\section{References}

Abdelraheem, A. (2013). Social networks sites: Usage and effects. Journal of Educational and Psychological Studies, 7(4), 549-558.

Abdulrahman, H. (2017). Emotional separation and its relationship with irrational thinking among married people. Unpublished Master Thesis, Yarmouk University, Irbid, Jordan.

Abu Asaad, A. (2008). Marital and family counseling. Al-Shorouk for Publishing.

Abu Dary, M. (2019). Jordan is the first in the world in the use of social media platforms. Addustour. Retrieved from: https://www. addustour.com/articles.

Abu Nada, A. (2007). Psychological hardness and its relationship to life stress among Al-Azhar University students in Gaza. Unpublished Master Thesis, Al-Azhar University, Gaza, Palestine.

Abu Zaid, N. (2011). Family psychology. Alamakotob for Publishing and Distribution.

Al-Ayafi, A. (2012). Psychological hardness and stressful life events among a sample of orphans and regular students in Makkah and Laith governorate. Unpublished Master Thesis, Umm Al-Qura University, Mecca, Saudi Arabia.

Al-Fatlawi, A., \& Jabbar, W. (2012). The emotional divorce and its relation with life styles of married employees in the government's offices. Al-Qadisiya Journal for Humanities, 15(1), 211-260.

Al-Furaiji, H. (2014). Social media: An educational jurisprudence view. Dar AlMahjah Al-Bayda.

Al-Ghurair, A., \& Abu Asaad, A. (2009). Stress coping. Dar Al-Shorouk for Publishing and Printing.

Al-Hamad, N., Al-Badarneh, M., \& Al-Momani, H. (2017). Psychological hardness and its relationship with the inability to psychological and academic adjustment among married and unmarried female students. Dirasat Humanities and Social Sciences, 44, 15-31.
Al-Jawazneh, B. (2018). The emotional divorce among couples and its impact on psychological adjustment of children in the secondary-stage families with broken families in Al-Karak governorate. Journal of Education, 18(1), 384-413.

Al-Mutawa, A. (2015), the impact of the WhatsApp network on some variables among a sample of married couples in the Saudi community. Journal of Scientific Research in Education, 3(61), 74-89.

Al-Obeid, M. (2014). Social media and its impact on social relations. Al-Hikma Journal, 2(6), 150- 187.

Al-Sadhan, A. (2013). Family guidance guide 6: The problem of emotional divorce and how the family guide deals with it. King Fahd National Library for Publishing.

Al-Shamrani, A. (2015). Social media networks' usage intensity and its relationship to the marital adjustment among teachers. Unpublished Master Thesis, Yarmouk University, Irbid, Jordan.

Al-Shehri, H. (2013). The effects of using electronic social networks on social relationships "facebook and twitter as examples: A survey study on a sample of King Abdul Aziz University Istudents in Jeddah. Unpublished Master Thesis, King Abdul Aziz University, Jeddah, Saudi Arabia.

Al-Smeheen, F. (2016). Marital adjustment and its relationship with psychological hardness and emotional maturity for married female teachers at the directorate of education of AlJamea district. Doctoral Dissertation, University of Jordan, Amman, Jordan.

Al-Taher, T. (2016). Psychological hardness. Delta College Journal of Science and Technology, University of El-Imam El-Mahdi, 4, 111-146.

Al-Tunbali, A. (2016). The impact of spousal addiction to the internet on marital expatriation from the perspective of general practice for social work. The Journal of Social Workers, 6(65), 17-20.

Al-Youssef, A. (2017). The impact of social media on family relationships in Irbid governorate, Unpublished Master Thesis, Yarmouk University, Irbid, Jordan. 
Al-Zoubi, M. (2014). Divorce and the procedures followed in the fatwa department to obtain a divorce fatwa. Retrieved from: https://www. aliftaa.jo/Research.aspx? ResearchId=73\#.XrpdGXVLIU .

Arab Social Media Report. (2015). Retrieved from: http://www.wpp.com.

Ashash, N. (2018). Methods of facing psychological stress and its relationship with psychological stiffness among university students. Journal of the Faculty of Education, Port Said University, 23, 401-430.

Askar, A. (2000). Stress of life and methods of confronting it: Mental and physical health in the era of anxiety. Dar Al-Kitab Al-Hadith.

Bartone, P., Kelly, D., \& Matthews, M. (2013). Psychological hardness predicts adaptability in military leaders: A prospective study. International Journal of Selection and Assessment, 21(2), 200-210.

Boumediene, Aajib. (2016), Family and social consequences of women working outside the home: A field study on a sample of women working in Laghouat. Doctoral Dissertation, University of Oran 2, Bir El-Jeer, Algeria.

Camellia, E. (2016). Psychology of working women. Dar AL-Nahda for Printing and Publishing.

Clarke-Stewart, A., \& Brentano, C. (2006). Divorce: Causes and consequences. Yale University Press.

Clayton, R., Nagourney, A., \& Smith, J. (2013). Cheating, breakup and divorce: Is facebook use to blame? Cyberpsychology, Behavior and Social Networking, 16(10), 717-720.

Crow, M. (2005). Overcoming relationship problems: A self-help guide using cognitive behavioral techniques. London: Constable and Robenson.

Fadlullah, W. (2010). Facebook impact on society. Khartoum: Shams Al-Nahda Blog.

Fahmi, D. (2018). Criminal protection from misuse of social media. Cairo: Dar Al-Nahda.

Fearon, J. (2003). Ethnic and cultural diversity by country. Journal of Economic Growth, 8(2), 195-222.
Ghazal, M., \& Shaobi, N. (2014). The impact of social media on the development of political awareness among university students. Unpublished Master Thesis, University Kasdi, Ouargla, Algeria.

Hadi, A. (2012). Reasons for emotional divorce among Iraqi families according to some variables. Al-Ustath Journal, 2(1), 435-462.

Hill, M., Stafford, A., Seaman, P., Ross, N., \& Daniel, B. (2007). Parenting and resilience. York: Joseph Rowntree Foundation.

Judkins, S. (2003). Hardness, stress and coping strategies among mid-level nurse managers: implications for continuing higher education. Doctoral Dissertation, University of North Texas, Denton.

Kasemi, N. (2012). Family sociology and social change. Dar Al-Kitab Al-Hadith.

Khoury, N. (2008). Marriage is a psychosocial approach. Lebanese Dar Al-Manhal for Publishing and Distribution.

Kumari, A., \& Verma, J. (2015). Impact of social networking sites on social interaction. International Journal of Humanities and Social Sciences, 4(2), 55-62.

Lambert, V., Lambert, C., \& Yamase, H. (2003). Psychological hardness, workplace stress and related stress-reduction strategies. Nursing \& Health Sciences, 5(2), 181-184.

Lkhal, H., \& Zaidi, R. (2017). The impact of using social media on family relationships: Facebook as a model. Master Thesis, University of Ziane Achour, Djelfa, Algeria.

Maddi, S. (2013). Hardness turning stressful circumstances into resilient growth. Springer: New York, London, Dordrecht, Heidelberg.

Mansour, A. (2009). Factors contributing to emotional separation among married couples and its consequences from the perspective of a sample of wives in Jordan. Doctoral Dissertation, Amman Arab University, Amman, Jordan.

Masoudan, A., \& Warim, E. (2012). Use of new media and communication and its relationship to social isolation: An analytical study of the impact of social networks on family communication. Education Journal - Al-Azhar University, 15(1), 739-767. 
Momen, D. (2004). Family and family therapy. ElSahab for Publishing.

Mukhaimar, I. (2002). Psychometric hardness scale. The Anglo-Egyptian Bookshop.

Najdawi, A. (2018). The view of women in emotional divorce: Applied study on a sample of Jordanian women. Dirasat: Human and Social Sciences, 45(2), 43-58.

Nejadat, A. (2014). The use of "Facebook" by married working women in Jordanian universities and its role in achieving satisfaction: A survey study. Jordan Journal of Social Sciences - Jordan, 7(1),103-131.

Odah, M. (2010). Traumatic experience and its relationship to the methods of adaptation to stress and social support and mental toughness to the children in the Gaza Strip border areas. Unpublished Master Thesis, Islamic University, Gaza, Palestine.

Parvin, S., Kalantari, A., Davoodi, M., \& Mohammadi, F. (2011). Emotional divorce in Tehran city. International Journal of Social Sciences, 1(4), 305-311.

Radcliffe, D., \& Bruni, P. (2019). State of social media: Middle East 2018. Researchgate. Retrieved from: https://www.researchgate.net/ publication/330842301_State of Social Media Middle East 2018.

Rady, Z. (2008). Psychological Hardness among the mothers of Al Aqsa Intifada martyrs and its relationship with some variables. Unpublished Master Thesis, The Islamic University, Gaza, Palestine.

Rahmani, N., \& Dhimi, Z. (2012). Adolescent internet education. Amman: Ibn Battuta House for Publishing.

Rodriguez, N., Smith, H., \& Zatz, M. S. (2009). Youth is enmeshed in a highly dysfunctional family system: Exploring the relationship among dysfunctional families, parental incarceration and juvenile court decisionmaking. Criminology, 47(1), 177-208.
Rosberg, G., \& Rosberg, B. (2003). Divorce-proof your marriage. Tyndale House Publishers, Inc.

Sadeq, A. (2004). Love without marriage and marriage without love. Dar Al-Sahwah for Publishing and Distribution.

Sedghijalal, A., \& Fathi, S. (2015). The virtual social networks and couples commitment. International Journal of Social Sciences, 5(3), 51-60.

Shakra, A. (2014). New media: Social media. Osama for Publishing and Distribution.

Shehata, M. (2015). An eclectic approach to alleviating the marital alienation of newly married internet users. Journal of Social Service: Association of Egyptian Specialists, 45, 331-377.

Shosha, N. (2015). The effect of communication on the disintegration of society and the family. Al-Bayan Magazine, issue 341 (October November). Retrieved from: http:// albayan. co.uk /MGZArticle2. aspx?ID=4673.

Shwaiter, K., \& Al-Zaqai, N. (2015). Psychological hardness among mothers working in the education sector in Oran: A descriptive psychometric study. Journal of Psychological and Educational Studies, 4, 47-66

Siddiqui, S., \& Singh, T. (2016). Social media: Its impact with positive and negative aspects. International Journal of Computer Applications, Technology and Research, 5(2), 71-75.

Subhi, S. (2016). The psychometric properties of psychological hardness scale among a sample of widows. Journal of Counseling Psychology, $48,252-267$.

Supreme Judge Department. (2017). Annual statistical report for 2017. Retrieved from: https: sjd.gov.jo/Pages/viewpage.aspx?pageID=206.

Theban, N. (2009). Divorce and marriage problems. Rislan for Publishing.

Yakhlof, O. (2001). Psychology of health psychological and behavioral bases of health. Dar Al Thaqafa. 